\title{
Mass and galaxy distributions of four massive galaxy clusters from Dark Energy Survey Science Verification data
}

\author{
P. Melchior, ${ }^{1,2 \star}$ E. Suchyta, ${ }^{2}$ E. Huff, ${ }^{1}$ M. Hirsch, ${ }^{3}$ T. Kacprzak, ${ }^{4}$ E. Rykoff, ${ }^{5}$ \\ D. Gruen, ${ }^{6,7}$ R. Armstrong, ${ }^{8}$ D. Bacon, ${ }^{9}$ K. Bechtol, ${ }^{10}$ G. M. Bernstein, ${ }^{8}$ S. Bridle, ${ }^{4}$ \\ J. Clampitt, ${ }^{8}$ K. Honscheid, ${ }^{2}$ B. Jain, ${ }^{8}$ S. Jouvel, ${ }^{3}$ E. Krause, ${ }^{8}$ H. Lin, ${ }^{11}$ \\ N. MacCrann, ${ }^{4}$ K. Patton, ${ }^{2}$ A. Plazas, ${ }^{12}$ B. Rowe,${ }^{3}$ V. Vikram, ${ }^{8}$ H. Wilcox,${ }^{9}$ \\ J. Young, ${ }^{6}$ J. Zuntz, ${ }^{4}$ T. Abbott,${ }^{13}$ F. B. Abdalla, ${ }^{3}$ S. S. Allam,,${ }^{11}{ }^{14}$ M. Banerji, ${ }^{3}$ \\ J. P. Bernstein, ${ }^{15}$ R. A. Bernstein, ${ }^{16}$ E. Bertin, ${ }^{17}$ E. Buckley-Geer, ${ }^{11}$ D. L. Burke, ${ }^{5,18}$ \\ F. J. Castander, ${ }^{19}$ L. N. da Costa, ${ }^{20,21}$ C. E. Cunha, ${ }^{18}$ D. L. Depoy, ${ }^{22}$ S. Desai, ${ }^{23,24}$ \\ H. T. Diehl, ${ }^{11}$ P. Doel, ${ }^{3}$ J. Estrada, ${ }^{11}$ A. E. Evrard, ${ }^{17,25,26}$ A. Fausti Neto, ${ }^{21}$ \\ E. Fernandez, ${ }^{27}$ D. A. Finley, ${ }^{11}$ B. Flaugher, ${ }^{11}$ J. A. Frieman, ${ }^{10,11}$ E. Gaztanaga, ${ }^{19}$ \\ D. Gerdes, ${ }^{25}$ R. A. Gruendl, ${ }^{28,29}$ G. R. Gutierrez, ${ }^{11}$ M. Jarvis,${ }^{8}$ I. Karliner,${ }^{30}$ S. Kent, ${ }^{11}$ \\ K. Kuehn, ${ }^{31}$ N. Kuropatkin, ${ }^{11}$ O. Lahav, ${ }^{3}$ M. A. G. Maia, ${ }^{20,21}$ M. Makler, ${ }^{32}$ \\ J. Marriner, ${ }^{11}$ J. L. Marshall, ${ }^{22}$ K. W. Merritt, ${ }^{11}$ C. J. Miller, ${ }^{26}$ R. Miquel, ${ }^{27,33}$ \\ J. Mohr, ${ }^{23}$ E. Neilsen, ${ }^{11}$ R. C. Nichol, ${ }^{9}$ B. D. Nord, ${ }^{11}$ K. Reil,${ }^{5,18}$ N. A. Roe, ${ }^{34}$ \\ A. Roodman, ${ }^{5,18}$ M. Sako, ${ }^{8}$ E. Sanchez, ${ }^{35}$ B. X. Santiago, ${ }^{21,36}$ R. Schindler, ${ }^{5,18}$ \\ M. Schubnell, ${ }^{25}$ I. Sevilla-Noarbe, ${ }^{35}$ E. Sheldon, ${ }^{12}$ C. Smith, ${ }^{13}$ M. Soares-Santos, ${ }^{11}$ \\ M. E. C. Swanson, ${ }^{29}$ A. J. Sypniewski, ${ }^{25}$ G. Tarle, ${ }^{25}$ J. Thaler,${ }^{30}$ D. Thomas,,${ }^{9,37}$ \\ D. L. Tucker, ${ }^{11}$ A. Walker, ${ }^{13}$ R. Wechsler, ${ }^{5,18}$ J. Weller ${ }^{6,7,24}$ and W. Wester ${ }^{11}$ \\ Affiliations are listed at the end of the paper
}

Accepted 2015 February 23. Received 2015 February 14; in original form 2014 May 16

\begin{abstract}
We measure the weak lensing masses and galaxy distributions of four massive galaxy clusters observed during the Science Verification phase of the Dark Energy Survey (DES). This pathfinder study is meant to (1) validate the Dark Energy Camera (DECam) imager for the task of measuring weak lensing shapes, and (2) utilize DECam's large field of view to map out the clusters and their environments over 90 arcmin. We conduct a series of rigorous tests on astrometry, photometry, image quality, point spread function (PSF) modelling, and shear measurement accuracy to single out flaws in the data and also to identify the optimal data processing steps and parameters. We find Science Verification data from DECam to be suitable for the lensing analysis described in this paper. The PSF is generally well behaved, but the modelling is rendered difficult by a flux-dependent PSF width and ellipticity. We employ photometric redshifts to distinguish between foreground and background galaxies, and a red-sequence cluster finder to provide cluster richness estimates and cluster-galaxy distributions. By fitting Navarro-Frenk-White profiles to the clusters in this study, we determine weak lensing masses that are in agreement with previous work. For Abell 3261, we provide the first estimates of
\end{abstract}


redshift, weak lensing mass, and richness. In addition, the cluster-galaxy distributions indicate the presence of filamentary structures attached to 1E 0657-56 and RXC J2248.7-4431, stretching out as far as $1^{\circ}$ (approximately $20 \mathrm{Mpc}$ ), showcasing the potential of DECam and DES for detailed studies of degree-scale features on the sky.

Key words: gravitational lensing: weak - galaxies: clusters: individual: RXC J2248.7-4431 galaxies: clusters: individual: 1E 0657-56-galaxies: clusters: individual: SCSO J233227-535827-galaxies: clusters: individual: Abell 3261-cosmology: observations.

\section{INTRODUCTION}

The Dark Energy Survey (DES) comprises an optical to nearinfrared survey over $5000 \mathrm{deg}^{2}$ of the South Galactic Cap to $\sim 24$ th mag in the Sloan Digital Sky Survey (SDSS) grizY bands and a time-domain griz survey over $30 \mathrm{deg}^{2}$ with a cadence of approximately $6 \mathrm{~d}$. These interleaved surveys are being carried out over 525 nights in the course of 5 yr using the 570-MP imager Dark Energy Camera (DECam; Diehl 2012; Flaugher et al. 2012) mounted at the prime focus of the Blanco 4-m telescope at NOAO's Cerro Tololo Inter-American Observatory. DECam was commissioned in 2012 September and October, followed by an extended testing and survey commissioning period known as DES Science Verification (SV; 2012 November-2013 February). With this new instrument, DES will go beyond the reach of SDSS by virtue of telescope aperture, median seeing, and CCD sensitivity, particularly towards the infrared part of the spectrum. Consequently, the galaxy redshift distribution is expected to have a median $z \approx 0.7$ and a significant tail beyond $z=1$, which enables DES to detect clusters at high redshift $(z \approx 1)$ and to use source galaxies for a rigorous lensing analysis of clusters beyond $z \approx 0.5$. DES will also exceed deep and medium-deep weak lensing surveys [Canada-FranceHawaii Telescope Legacy Survey (CFHTLS), Red-sequence Cluster Survey 2 (RCS2), Deep Lens Survey (DLS)] by up to an order of magnitude in area. More details about the survey can be found in The Dark Energy Survey Collaboration (2005) and at http://www.darkenergysurvey.org.

The very wide field of view of DECam of slightly more than $3 \mathrm{deg}^{2}$ allows us to capture the environment of even the most massive galaxy clusters with a single pointing. In this paper we will show results on four fields containing clusters with masses of approximately $1-2 \times 10^{15} \mathrm{M}_{\odot}$ and redshifts from $z=0.22$ to 0.40 . They have been chosen to demonstrate the capabilities of DECam imaging for cluster and lensing analyses and provide an outlook of the utility of the entire DES to map out a good fraction of the sky to redshifts of about 1 .

The goals of this pathfinder study are twofold. First, to validate the data quality delivered by DECam for the purpose of galaxy cluster and lensing studies. We focus our attention on four fields imaged during the SV period in grizY filters with integration times characteristic of the DES to study the relevant elements of photometry and image quality. We inspect our ability to model the point spread function (PSF) and to account for possible systematic contaminants of photometry and lensing analyses. We emphasize that this is not a comprehensive study of the DES pipelines for photometry or lensing; those studies will be presented elsewhere.

Second, we want to utilize the large field of view of DECam to map the environments of these clusters over 90 arcmin, probing clustercentric distances between 10 and $15 \mathrm{Mpc}$ at the respective cluster redshifts. We select background galaxies according to their photo- $z$ estimates, use the red-sequence cluster finder redMaPPer to identify cluster galaxies, and employ IM3SHAPE for weak lensing shape measurements, from which we obtain mass estimates and two-dimensional mass maps.

Section 2 contains the details of the observations and data reduction pipeline used in this study. Section 3 describes the photometric calibration (Section 3.1), the redMaPPer technique for identifying red-sequence galaxies (Section 3.2), the photometric redshift methodology (Section 3.3), and the background galaxy selection procedure (Section 3.4). Section 4 describes the lensing analysis, detailing PSF estimation (Section 4.1), shape measurements with IM3SHAPE (Section 4.2), and the combination of measurements in three bands into a single shape catalogue (Section 4.3). We perform additional tests of the recovered cluster shears in Section 4.4 and present the Navarro-Frenk-White (NFW) profile fits and lensing mass estimates in Section 5.1 and the mass-richness relation in Section 5.2. We show mass and cluster galaxy maps in Section 6 and indications for the presence of filamentary structures in two of the investigated fields (Section 6.2). We summarize our findings in Section 7.

For the entire paper we adopt a flat $\Lambda$ cold dark matter $(\Lambda \mathrm{CDM})$ cosmological model with $\Omega_{\mathrm{m}}=0.3$ and $H_{0}=100 \mathrm{hm} \mathrm{s}^{-1} \mathrm{Mpc}^{-1}$, where $h=0.7$.

\section{OBSERVATIONS AND DATA PROCESSING}

The clusters targeted for this study are both massive and at intermediate redshift so as to show up prominently in our weak lensing measurements. In detail, we targeted 1E 0657-56 (known as the Bullet cluster; Tucker et al. 1998), RXC J2248.7-4431 (Böhringer et al. 2004), and SCSO J233227-535827 (Menanteau et al. 2010a). ${ }^{1}$ All of these systems are well studied, providing us with important information such as spectroscopic redshifts and mass estimates from lensing or baryonic tracers. General properties of the clusters are listed in Table 1.

The exposures for these clusters were taken over the course of several nights (2012 November 16-24 and 2012 December 7). We adhered to the nominal DES exposure times: $90 \mathrm{~s}$ in $g, r, i, z$ and $50 \mathrm{~s}$ in $Y$, and used a 10-exposure dither pattern centred on the cluster with offsets of around 0.1 . Hence, the total depth of these observations is characteristic of the DES main survey, but differs in the dither pattern.

We extended the data set in two ways. First, we re-observed one cluster, RXC J2248.7-4431, later in the season (on 2013 August 15) to benefit from improvements to telescope performance and

\footnotetext{
${ }^{1}$ In the course of this programme, we also observed ACT-CL J0102-4915 (dubbed El Gordo; Menanteau et al. 2010b), but the images are rather shallow for a dedicated weak lensing analysis of this cluster at $z=0.87$. We therefore omit the cluster in this work.
} 
Table 1. The cluster sample. Coordinates correspond to the centroids of the BCGs, redshifts are spectroscopically confirmed. The labels are used as abbreviations throughout this work.

\begin{tabular}{llllc}
\hline Cluster name & Label & RA $\left(^{\circ}\right)$ & Dec. $\left(^{\circ}\right)$ & $z$ \\
\hline RXC J2248.7-4431 & RXJ & 342.18319 & -44.53091 & 0.348 \\
1E 0657-56 & Bullet & 104.64708 & -55.94897 & 0.296 \\
SCSO J233227-535827 & SPTW1 & 353.11446 & -53.97441 & 0.402 \\
Abell 3261 & Abell 3261 & 67.31375 & -60.32555 & - \\
\hline
\end{tabular}

general image quality. Second, in order to compare our findings from the targeted cluster fields to typical DES survey performance, we added another cluster, Abell 3261 (Abell, Corwin \& Olowin 1989), to our investigation. It was observed during tests of survey operations, when DES observations are coordinated by an automated observer tactician program, OBSTAC (Neilsen \& Annis 2014), which selects upcoming exposures based upon survey history and current conditions. ${ }^{2}$ Thus, for this cluster the imaging data experience seeing conditions, sky brightness levels, and the dither pattern typical of the main survey.

All raw data presented here, including the calibration images, are public and can be obtained from the National Optical Astronomy Observatory (NOAO) archive.

\subsection{Observational conditions}

The conditions during the observations were generally stable and mostly photometric. During the November runs, the moon illumination was bright enough to significantly reduce the depth in $g$ and $r$, triggering re-observations during dark conditions on December 7. The seeing in these nights varied between 0.8 and $1.0 \operatorname{arcsec}$ in $i$, with larger seeing values in $r$ and particularly $g$, in agreement with atmospheric turbulence models. The stellar ellipticity was typically smooth across the field of view, ${ }^{3}$ and varied only slowly with time during the same night, indicating that the PSF is dominated by the optics rather than the atmosphere. If observations spanned multiple nights, non-trivial variations occurred, which render the PSF modelling more challenging. The observations from November exhibit a predominant elongation in the right ascension direction due to telescope tracking oscillations. Because of mechanical improvements of the telescope between these first sets of exposures and the re-observations on December 7 and later during the SV period, the latter ones show smaller overall ellipticity and can be well described by the typical optical aberrations of a wide-field imager. An overview of average seeing conditions for all data in question is given in Table 2 .

\subsection{Co-add images and catalogues}

Our treatment of the data begins with single-epoch images that have been processed through the DES Data Management (DESDM) pipeline (Desai et al. 2012; Mohr et al. 2012). Because a significant portion of our observations was taken early in the SV program, we

\footnotetext{
${ }^{2}$ One key decision made by OBsTAC is to observe in the $r, i, z$ bands only if the seeing in the proposed band is better than $1.1 \mathrm{arcsec}$, taking into account the chromatic and airmass dependence.

${ }^{3}$ To the degree we could determine from our observations, which comprise the Bullet cluster field that exhibits a strongly enhanced stellar density due to its low galactic latitude. However, dedicated studies on star clusters were not performed.
}

Table 2. Average PSF width (seeing) and ellipticity for each filter in each of the four fields, with re-observations indicated by a running number. Observations marked with $\uparrow$ were completed in the following night; those marked with $\ddagger$ were taken under OBSTAC control during survey mode operations between 2012 December and 2013 January 2013. Seeing is given in terms of the FWHM in arcsec, the ellipticity in terms of shears, not polarizations. The last column 'Shapes' denotes whether the co-add image was suitable for a weak lensing shape analysis (see Section 4.1.2 for details on the selection).

\begin{tabular}{lllccc}
\hline Field & Band & Date & Seeing & Ellipticity & Shapes \\
\hline Bullet & $g$ & $2012-12-07$ & 1.06 & 0.038 & \\
Bullet & $r .1$ & $2012-11-23 \dagger$ & 1.04 & 0.056 & $\sqrt{ }$ \\
Bullet & $r .2$ & $2012-12-07$ & 0.93 & 0.027 & $\sqrt{ }$ \\
Bullet & $i$ & $2012-11-23 \dagger$ & 1.00 & 0.032 & $\sqrt{ }$ \\
Bullet & $z$ & $2012-11-23 \dagger$ & 0.97 & 0.039 & $\sqrt{ }$ \\
Bullet & $Y$ & $2012-11-23 \dagger$ & 1.00 & 0.047 & \\
RXJ & $g$ & $2012-12-07$ & 1.18 & 0.031 & \\
RXJ & $r .1$ & $2012-11-24$ & 0.92 & 0.041 & $\sqrt{ }$ \\
RXJ & $r .2$ & $2012-12-07$ & 1.08 & 0.019 & $\sqrt{ }$ \\
RXJ & $i .1$ & $2012-11-24$ & 0.86 & 0.029 & \\
RXJ & $i .2$ & $2013-08-15$ & 0.79 & 0.023 & $\sqrt{ }$ \\
RXJ & $z .1$ & $2012-11-24$ & 0.90 & 0.045 & \\
RXJ & $z .2$ & $2013-08-15$ & 0.76 & 0.027 & $\sqrt{ }$ \\
RXJ & $Y$ & $2012-11-24$ & 0.85 & 0.042 & \\
SPTW1 & $g .1$ & $2012-11-16 \dagger$ & 1.4 & 0.025 & \\
SPTW1 & $g .2$ & $2012-12-07$ & 1.13 & 0.037 & \\
SPTW1 & $r$ & $2012-11-17$ & 0.97 & 0.027 & $\sqrt{ }$ \\
SPTW1 & $i$ & $2012-11-18$ & 0.99 & 0.036 & $\sqrt{ }$ \\
SPTW1 & $z$ & $2012-11-17$ & 0.90 & 0.029 & $\sqrt{ }$ \\
SPTW1 & $Y$ & $2012-11-16$ & 1.15 & 0.029 & \\
Abell 3261 & $g$ & $\ddagger$ & 1.10 & 0.024 & \\
Abell 3261 & $r$ & $\ddagger$ & 0.95 & 0.026 & $\sqrt{ }$ \\
Abell 3261 & $i$ & $\ddagger$ & 0.87 & 0.016 & $\sqrt{ }$ \\
Abell 3261 & $z$ & $\ddagger$ & 1.03 & 0.024 & $\sqrt{ }$ \\
Abell 3261 & $Y$ & $\ddagger$ & 0.87 & 0.028 & \\
\hline
\end{tabular}

first visually inspected our images for adequate data quality and rejected problematic exposures, e.g. those affected by occasional guiding failures. We excluded symptomatic frames, characterized by elongated, distorted stellar profiles. In a few instances we also rejected exposures showing significant levels of scattered light across the focal plane.

The DESDM pipeline already implements several standard detrending corrections for the raw data. The overscan is subtracted and a cross-talk matrix removes effects that bright stars induce across the detector. Bias frames and dome flats are averaged over several nights and then applied to the data. Included in the DESDM reductions are fringe and pupil-ghost corrections as well as the photometric calibration, which we detail in Section 3.1.

Astrometric solutions are computed by DESDM using SCAMP (Bertin 2006), matching absolute stellar positions in each exposure separately to the UCAC4 reference catalogue (Zacharias et al. 2013). While we do not require precise absolute astrometry, the relative astrometry between individual exposures is critical for accurate shape measurements. We therefore re-run SCAMP, simultaneously matching all filters and exposures to each other as well as to the much sparser reference catalogue. Typical errors after this step are 5 mas shifts between bands and 20 mas scatter within a band, improving the single-epoch solutions by factors of $\sim 3$.

We then co-add the single-epoch images of each $g, r, i, z, Y$ filter band separately. Additionally, we combine the bands considered for shape measurement into a multiband riz co-add for use as a 


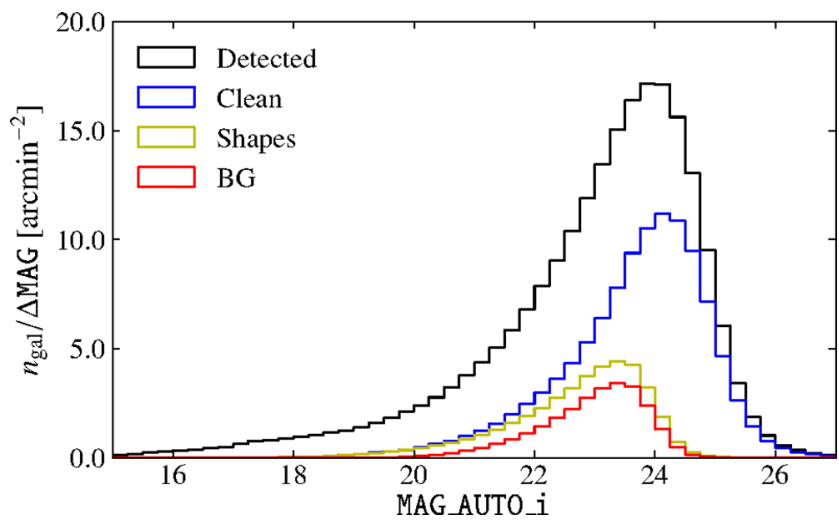

Figure 1. Galaxy number density $n_{\text {gal }}$ in bins of SExTRACTOR's magnitude MAG_AUTO in the $i$ band. From top to bottom: detected sources (black); after cleaning the catalogue (blue); galaxies with successful shape measurements (yellow); galaxies considered in the lensed background sample (red). See Section 4.3 for details on the selections. The numbers are unweighted, averaged over all four fields.

source detection image of increased depth and redshift coverage. Co-addition is implemented with SW ARP (Bertin et al. 2002), using a clipped mean algorithm we have added to the software (Gruen, Seitz \& Bernstein 2014a), which rejects pixels that are outliers at the $4 \sigma$ level. ${ }^{4}$ During co-addition, appropriate flux scalings are applied to each image using the zero-points calculated by the DESDM photometric calibrations.

We run SExtractor (Bertin \& Arnouts 1996) in dual-image mode: we provide the riz co-add for detection of sources and assignment of pixels to detections, afterwards the photometric measurements are made from the single-band values in these pixels. The detection threshold is set to $1.5 \sigma$ above noise in at least six contiguous pixels. The magnitude distribution of detected sources can be seen in Fig. 1.

Finally, we define a field mask to restrict the analysis to full-depth areas, excluding the edges of each pointed field (RXJ, Bullet, and SPTW1). We decided on a square shape with a side length of 1.5 with trimmed corners (shown in the two left-hand panels of Fig. 3), the centre of which was adjusted so as to maximize the number of total detections.

\section{PHOTOMETRY AND GALAXY SAMPLE SELECTION}

In this work, the major role of photometry is to separate galaxies by colour and to assign photometric redshifts to them for proper calculation of cosmological distances and lensing weight factors (Section 4.3). We will therefore only introduce the general photometric approach, focusing on aspects most relevant for this study and discussing the accuracy with which we can perform these photometric tasks, and refer the reader to forthcoming publications for details.

\subsection{Photometric calibration}

Each individual exposure is first processed to bring all pixels of the array on to a common photometric scale. Details are given in

\footnotetext{
${ }^{4}$ In contrast to median co-adds, this approach successfully rejects cosmic ray hits without sacrificing image fidelity and statistical optimality or altering the PSF shape, critical for weak lensing studies.
}

Tucker et al. (2007), so we only summarize the main aspects here. After bias subtraction, the images are divided by a dome flat. The dome flat is known, however, to have variations due to stray light and to changes in the effective pixel area. To reduce the impact of these variations on the object photometry we divide the images by a star flat (e.g. Manfroid 1995), which provides a correction for $512 \times 512$ pixel regions on each CCD (32 regions per CCD) that minimizes the dependence of the stellar photometry on the DECam focal plane position.

Exposures taken on clear nights are brought to a common absolute magnitude scale by using a zero-point that is a linear function of airmass, with slope and offset fit to observations of SDSS photometric standard fields taken at the beginning and end of the night. At least one photometric exposure is required for each filter in each field. We use these observations to create a local set of standard stars, for each field, by averaging over all overlapping objects. We then refit all CCD zero-points from both photometric and non-photometric conditions by allowing each DECam CCD on each exposure to have its own zero-point. Zero-points are adjusted to minimize the difference in magnitude between observations of common objects in different exposures and observations of the aforementioned local standards. This method typically gives an rms accuracy of $\sim 1$ per cent and has been validated by comparing measurements of the stellar locus in colour-colour space, as described below.

Stellar locus regression (SLR) has proven to be a useful complementary photometric calibration method to standard star observations, and relies upon the approximate universality of the intrinsic colours of Milky Way halo stars as a population (e.g. High et al. 2009). In the SLR approach, zero-points for each filter are adjusted until the foreground stars lie along the expected colour-colour locus. Since the great majority of stars detected in our DECam images are located beyond the Galactic dust sheet, the SLR is sensitive to the combined effects of atmospheric and interstellar extinction.

Our SLR implementation employs the publically available BIG MACS ${ }^{5}$ code developed by Kelly et al. (2014). The reference stellar locus is synthetically generated using the Pickles (1998) stellar spectroscopic library spliced with SDSS spectra (as described in Kelly et al. 2014) and convolved with the DECam total system transmission functions. Stars in the cluster fields are selected by requiring SEXTRACTOR's stellarity parameter CLASS_STAR $>0.95$ in both the $r$ and $i$ band, which provides adequate star-galaxy separation for high signal-to-noise ratio objects. Accordingly, we also require magnitude uncertainties of $<0.05(<0.1)$ in the $r i(g z)$ bands. In order to evaluate the photometric calibration across each cluster field, stars are binned into HEALPix ${ }^{6}$ (Górski et al. 2005) pixels with a resolution of $\sim 14 \operatorname{arcmin}(\mathrm{NSIDE}=256) .{ }^{7}$ We then allow the zero-points of the DECam griz filters to float independently in each spatial pixel during the fits and use the $J$-band magnitudes of matched Two Micron All Sky Survey (2MASS) stars for absolute calibration. The zero-point shifts fitted via SLR are typically $\lesssim 0.05 \mathrm{mag}$, with an associated statistical precision of $\sim 0.02$ mag estimated via bootstrapping of the stellar sample. The exception is the Bullet cluster field, where the median $g$-band zero-point shift is $\sim 0.2 \mathrm{mag}$, consistent with interstellar extinction expected in this low-Galactic-latitude field from the dust maps produced by Schlegel, Finkbeiner \& Davis (1998).

\footnotetext{
${ }^{5}$ http://code.google.com/p/big-macs-calibrate/

${ }^{6}$ http://healpix.sourceforge.net/

7 The choice of 14 arcmin constitutes a compromise between increasing angular resolution and maintaining a sufficient number of stars in each pixel such that statistical uncertainties are $\sim 0.02 \mathrm{mag}$.
} 


\subsection{Cluster member selection}

Our study uses the methodology from the red-sequence matched filter probabilistic percolation ('redMaPPer') algorithm (Rykoff et al. 2014), based on the optimized richness estimator $\lambda$ (Rykoff et al. 2012). redMaPPer is a photometric cluster finder that identifies galaxy clusters as overdensities of red-sequence galaxies, and has been shown to have excellent performance in photo- $z$ determination, purity, and completeness for wide-field photometric surveys (Rozo \& Rykoff 2014). The algorithm is divided into two stages: the first is a calibration stage where the red-sequence model is derived directly from the data, and the second is the cluster-finding stage. These two stages are iterated several times before a final cluster-finding run is performed.

In the calibration phase, redMaPPer empirically calibrates the colour distribution (mean and scatter) of red-sequence galaxies as a function of redshift and magnitude. For the red-sequence calibration, 356 spectroscopically confirmed brightest cluster galaxies (BCGs) in the 241-deg ${ }^{2}$ griz DES SV-A1 galaxy catalogue (Rykoff et al., in preparation) were used. The spectroscopic redshifts were taken from SDSS Data Release 10 (DR10; Ahn et al. 2014), South Pole Telescope (SPT) clusters (High et al. 2010), and as part of the OzDES programme (Lidman et al., in preparation). These galaxies are used as 'seeds' to look for significant overdensities of nearby galaxies with similar colour as the seed galaxy $(g-r, r-i$, or $i$ $-z$ depending on the redshift, as determined from MAG_DETMODEL ${ }^{8}$ magnitudes). The resulting set of cluster galaxies is used to fit a full red-sequence model including zero-point, tilt, and scatter. This scatter is characterized by a full covariance matrix among all colours (see Rykoff et al. 2014 for details). The red-sequence model is calibrated down to a luminosity threshold of $0.2 L_{*}$ at the cluster redshift, which was determined to be the optimal depth for cluster richness estimation (Rykoff et al. 2012). In this way we leverage the bright spectroscopic sample to obtain a model of the red sequence that extends to faint magnitudes.

Given the red-sequence model and the corrected magnitudes, the cluster-finding proceeds as follows. First, we consider all photometric galaxies as candidate cluster centres. The red-sequence model is used to calculate a photometric redshift for each galaxy, and evaluate the goodness of fit of our red-sequence template. Galaxies that are not a reasonable fit to the model at any redshift are immediately discarded. For the remaining galaxies, we use this initial redshift guess to evaluate the richness $\lambda$ and the total cluster likelihood. When at least three red-sequence galaxies (brighter than $0.2 L_{*}$ within a scale radius $r_{\lambda}$ ) are detected, we re-estimate the cluster redshift by performing a simultaneous fit of all the high-probability cluster members to the red-sequence model. This procedure is iterated until convergence is achieved between member selection and cluster photometric redshift, denoted $z_{\lambda}$. The resulting list of candidate cluster centres is then rank-ordered according to likelihood, and membership probabilities are used to mask out member galaxies in the final percolation step. All richness values are corrected for variations in the local depth of the DES imaging (Rykoff et al., in preparation), however, the DES imaging is deep enough that this has a negligible effect at the redshifts of the clusters considered in this paper. We have shown that for $\lambda>20$ the cluster purity and completeness are $>95$ per cent (Rozo \& Rykoff 2014; Rykoff et al. 2014). In addition, even for $\lambda>5$ the photo- $z$ performance is very

\footnotetext{
${ }^{8}$ In SExTRACTOR's dual-image mode, MAG_DETMODEL measures the flux in each filter by adopting a model fit to the object in the detection image, in our case the riz co-add.
}

good, with a scatter $\sigma_{z}<0.015$ for SV-A1 data (Rykoff et al., in preparation).

\subsection{Photometric redshifts}

We compute photometric redshifts (photo- $z$ s) using the artificial neural network method that was applied to the SDSS Data Release 6 (DR6) sample, as described in detail by Oyaizu et al. (2008a). In brief, we use a neural network configuration with 10 input nodes, consisting of five griz,Y MAG_AUTO magnitudes and five griz $Y$ MAG_DETMODEL magnitudes, followed by three hidden layers with 15 nodes layer ${ }^{-1}$. The neural network was trained using a set of about 12000 galaxies with DES main-survey depth photometry and high-confidence spectroscopic redshifts.

The accompanying photo- $z$ errors are computed using the empirical 'nearest neighbour error' (NNE) technique, described in detail by Oyaizu et al. (2008b). The NNE method estimates the photo- $z$ error for each galaxy empirically, based on the photo-zs and redshifts of the galaxy's 100 nearest neighbours in the spectroscopic validation sample, where neighbour distance is defined using a simple flat metric in the space consisting of the 10 input magnitudes noted above. Specifically, the NNE photo- $z$ error $\Delta z_{\text {phot }}$ is defined so that it corresponds to 68 per cent of the $\left|z_{\text {phot }}-z_{\text {spec }}\right|$ distribution of the nearest neighbours. In DES comparison tests by Sánchez et al. (2014), where the method we employ here is called DESDM, it shows a marginal bias $\langle\Delta z\rangle=-0.005 \pm 0.003$, a scatter of $\sigma_{68}=0.094 \pm 0.002$, and a $3 \sigma$ outlier rate of $0.018 \pm 0.003$. All of these metrics indicate that the photo- $z$ accuracy is easily sufficient for the purpose of this work. For further details, we refer the reader to Sánchez et al. (2014).

The photo- $z$ s also serve us to estimate

$\Sigma_{\mathrm{crit}}=\frac{c^{2}}{4 \pi G} \frac{D_{\mathrm{s}}}{D_{1} D_{\mathrm{ls}}}$,

which will be used below to relate the gravitational shear $\gamma$ to the surface density contrast of the cluster lens

$\Delta \Sigma=\Sigma_{\text {crit }} \gamma$.

In equation (1), $D$ denotes angular diameter distance: to a source at photometric redshift $z_{\text {phot }}$, to the lens at the spectroscopic redshift $z_{1}$ (cf. Table 1; for Abell 3261, we adopt the redMaPPer estimate from Table 4), and between lens and source.

\subsection{Background galaxy selection}

We select background galaxies according to their photometric redshifts $z_{\text {phot }}$ by requiring that

$z_{\text {phot }}>z_{1}+0.2$.

This seemingly straightforward selection is very effective at selecting a sample of galaxies that are behind the cluster, but due to the finite accuracy of the photo- $z$ s it is not perfect. Two main limitations need to be addressed and are discussed below.

\subsubsection{Cluster-member contamination}

Even though we reject all redMaPPer-detected galaxies in groups close to $z_{1}$ from the background sample, we find that the number density of nominally background galaxies, for which we have both a photo- $z$ and a shape estimate (see Section 4.3 for details), rises strongly towards the cluster centres (cf. Fig. 2). The rise is 


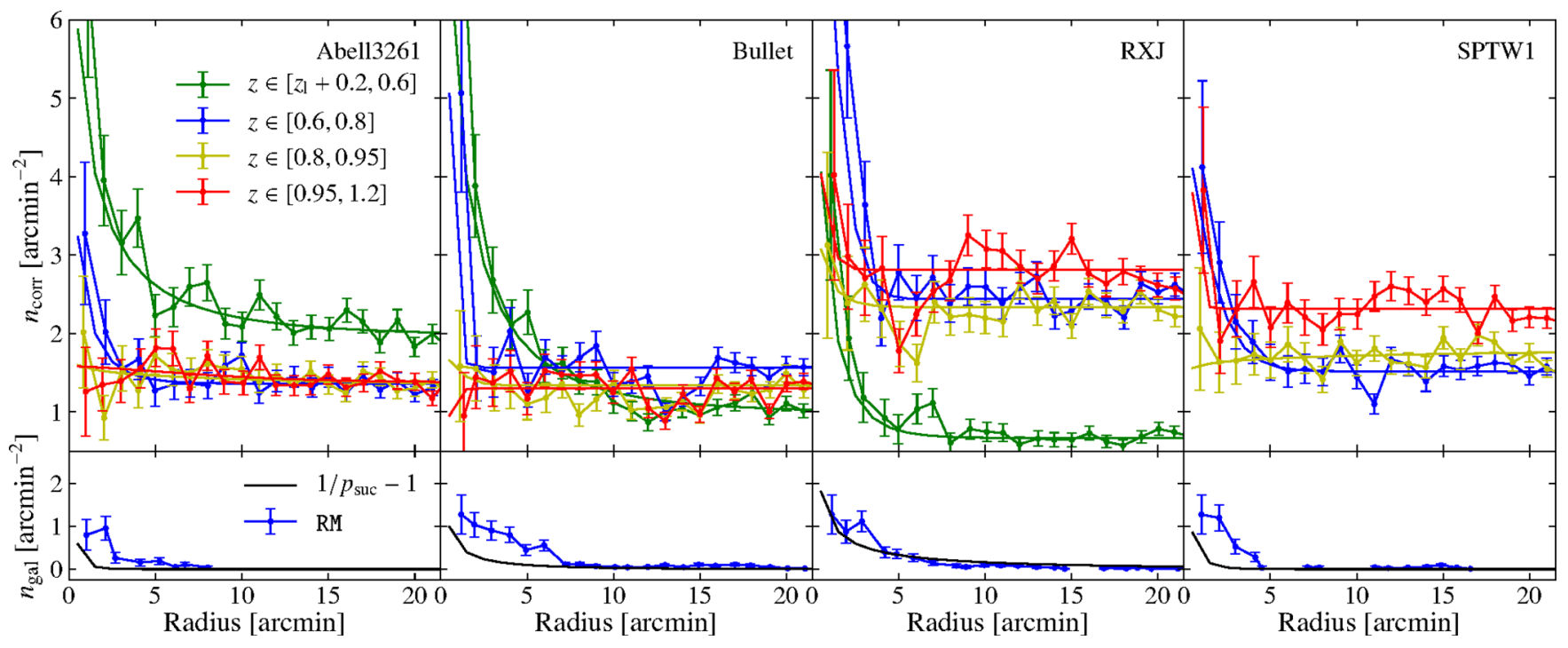

Figure 2. Top: number density of galaxies with shape measurements (cf. Section 4.2) in several photo- $z$ slices for each of the cluster fields. The solid lines are exponential fits to the corrected BG sample density (cf. equation 4 and Fig. 1). Bottom: redMaPPer-detected red-sequence galaxies in a narrow range around the cluster redshift (blue), and the measured success-rate corrections $1 / p_{\text {suc }}-1$ (black), applied to the number densities shown above as per equation (6).

caused by cluster member galaxies, for which neither redMaPPer nor the photo- $z \mathrm{~s}$ are complete or accurate enough to put them at the cluster redshift. As expected, the chance of such galaxies getting upscattered to a particular redshift drops with increasing separation between $z_{1}$ and $z_{\text {phot }}$.

Like Applegate et al. (2014), we correct for this effect by fitting an exponential model to the galactic number density as a function of clustercentric distance $r$ :

$n_{\text {corr }}(r)=n_{0}\left[1+\delta n_{C, Z} \exp \left[-\left(\frac{r}{r_{C, Z}}\right)^{\alpha_{C, Z}}\right]\right]$,

where $\delta n_{C, Z}, r_{C, Z}$, and $\alpha_{C, Z}$ are free parameters and allowed to differ for each cluster $C$ and each photo- $z$ slice $Z .^{9}$ The slices are chosen to have a roughly constant and sufficient number of galaxies to allow a successful fit. The resulting fits are shown as solid lines in Fig. 2. If we assume all of these contaminating galaxies to be randomly oriented, ${ }^{10}$ their effect is to reduce the perceived lensing effect of the background sample proportional to the contamination fraction. Following Blazek et al. (2012), we can therefore absorb the correction term into

$\Sigma_{\text {crit }, C, Z}=\Sigma_{\text {crit }}\left[1+\delta n_{C, Z} \exp \left[-\left(\frac{r}{r_{C, Z}}\right)^{\alpha_{C, Z}}\right]\right]$.

Note that $\Sigma_{\text {crit }}$ still depends on the actual (central) value $z_{\text {phot }}$ of each source, but the correction term is necessarily averaged over photo- $z$ slices of $\sim 0.2$ width. Because the contamination fraction quickly rises when $z_{\text {phot }} \rightarrow z_{1}$, we expect that this best effort correction is not entirely accurate, specifically for the lowest photo- $z$ slices.

As detailed in Applegate et al. (2014), to obtain a meaningful $n_{\text {corr }}$, one has to account for all effects that could change the number density, either related to the cluster or otherwise. First, masks around bright stars reduce the number of observable galaxies. At large

\footnotetext{
${ }^{9}$ If any form of additional weighting is employed (as we will do with equation 15 later on), $n_{0}$ needs to refer to the weighted number density.

${ }^{10}$ Intrinsic alignments, in particular in combination with photo- $z$ errors, could introduce non-random contributions to the lensing signal, but their strengths have been found to be insignificant for the work presented here (e.g. Chisari et al. 2014; Sifón et al. 2015).
}

distances, their impact is averaged out and only affects $n_{0}$. At smaller distance, we excised the radial range with prominent stars from the fit. This was necessary in the Bullet cluster field between 10 and 15 arcmin and in the RXJ field between 4 and 7 arcmin.

Second, the lensing-induced magnification could alter the observable number of galaxies, depending on the faint-end slope of the luminosity function. Even for clusters as massive as this, the magnification effect on the number counts is prominent only at small clustercentric distances, which we will exclude from our lensing mass estimates in Section 5.1, and is thus left uncorrected.

Third, the high density of large galaxies in the core region of clusters prevents us from detecting background galaxies or measuring their shapes accurately. In other words, the success fraction of shape measurements declines towards the centre, which could hide a substantial cluster-member contamination for the sample of galaxies we can measure shapes of. We assess the success probability $p_{\text {suc }}(r)$ with the newly developed code BALROG ${ }^{11}$ that allows us to insert artificial galaxies into our co-add images and compare the resulting catalogues with the input catalogues (Huff et al., in preparation) . In particular, we perform object detection and shape measurement, including any additional cuts, identically to the actual data analysis and then infer the rate of galaxies with acceptable shapes as a function of clustercentric distance. Since we can only count galaxies after their numbers $n_{\text {gal }}$ have been reduced due to blending with the cluster galaxies, we need to correct for the effect according to

$n_{\text {corr }}(r)=\frac{n_{\text {gal }}(r)}{p_{\text {suc }}(r)}$,

which brings these numbers, and hence the parameters of the fit in equation (4), back to values they would have without the presence of cluster-member galaxies. The dimensionless term $1 / p_{\text {suc }}-1$ is shown for each clusters as black line in the bottom panels of Fig. 2, where we contrast it with the number density of redMaPPer-detected galaxies (blue lines). We take the steeper profile of the $p_{\text {suc }}$ boost factor as an indication that the main difficulty for measuring shapes

\footnotetext{
${ }^{11}$ https://github.com/emhuff/Balrog
} 
in the central cluster regions stems from the existence of very large cluster galaxies, foremost the BCG, not just their increased number density.

We remark that the approach outlined here is very similar to the treatment in Applegate et al. (2014), in that the measured number densities are used to estimate the cluster-member contamination. But where they had to resort to proxies to estimate the success rate of their shape measurements, we actually measure $p_{\text {suc }}$ directly from our images.

\subsubsection{Photo-z inaccuracies}

The redshift estimate $z_{\text {phot }}$ still enters directly into $\Sigma_{\text {crit }}$, effectively treating $z_{\text {phot }}$ as the true redshift of the source $z_{\mathrm{s}}$. This is wrong in three ways. First, $\Sigma_{\text {crit }}$ is a non-linear function of $z_{\mathrm{s}}$, therefore, even symmetric uncertainties $\Delta z_{\text {phot }}$ as estimated in Section 3.3 lead to biased results for $\Sigma_{\text {crit }}$ and thus for $\Delta \Sigma$, the net effect of which is an underestimate of $\Sigma_{\text {crit }}$ that strongly rises with $z_{\text {phot }} \rightarrow$ $z_{1}$. Second, one can furthermore imagine that occasionally $\Delta z_{\text {phot }}$ $>0.2$, so that galaxies that are actually in front of the cluster make it into our background sample defined by equation (3). And third, the estimated photo- $z \mathrm{~s}$ (or their errors) could be catastrophically wrong, so that we may misestimate $\Sigma_{\text {crit }}$ with consequences yet to be determined.

We make use of the spectroscopic reference sample again and compute the true redshift distribution $p\left(Z_{\mathrm{s}} \mid z_{\text {phot }} \in Z\right)$ of sources in photo- $z$ slices $Z$ and spectroscopic slices $Z_{\mathrm{s}}$ of width 0.1 . Adopting a strategy closely related to Blazek et al. (2012), we determine the correction factor

$c_{Z}^{-1}=\frac{\sum_{Z_{\mathrm{s}}} p\left(Z_{\mathrm{s}} \mid z_{\text {phot }} \in Z\right) \Sigma_{\text {crit }}^{-1}\left(Z_{\mathrm{s}}\right)}{\Sigma_{\text {crit }}^{-1}(Z)}$,

whereby we mean $\Sigma_{\text {crit }}(Z)=\bar{\Sigma}_{\text {crit }}\left(z_{\text {phot }} \in Z\right)$, and likewise for the spectroscopic slice $Z_{\mathrm{s}}$. Applying this correction,

$\Sigma_{\text {crit }, C, Z, Z_{\mathrm{s}}}=c_{Z} \Sigma_{\text {crit }, C, Z}$,

we control for the three photo- $z$ errors on $\Delta \Sigma$ mentioned above at the level of $\Delta z_{\mathrm{s}}=0.1$, which is more fine grained than our cluster-member contamination correction from Section 3.4.1. For the remainder of this work, we will only work with corrected $\Sigma_{\text {crit }}$ values according to equation (8) without specifying it explicitly. A quantitative assessment of the amplitude of all corrections introduced in Section 3.4 is given at the end of Section 5.1.

\section{WEAK LENSING ANALYSIS}

For this paper, we adopt a shape measurement strategy, in which we perform the analysis on single-filter co-adds and combine the results from the $r, i, z$ filters at the ellipticity level. While neither statistically nor systematically optimal, ${ }^{12}$ working with co-added images, commonly done in cluster lensing studies, allows us to perform the shape measurements - of both stars and galaxies - at relatively high significance, the importance of which will become evident in the next section.

\footnotetext{
${ }^{12}$ The potential errors of the image co-addition could be avoided if the shape measurements are done on single-epoch images (cf. Miller et al. 2013), provided that the PSF can be modelled well with stars of lower significance. One can also reduce the noise-induced shape measurement biases by performing simultaneous shape fitting of all available exposures, even across filters. Both of these improvements are pursued for future lensing analyses in DES but go beyond the scope of this paper.
}

\subsection{PSF modelling}

Ordinarily, one models the PSF and its spatial variations simply by building a model from all available stars in the field. Unfortunately, PSF modelling for DECam is somewhat more complicated because its thick deep-depleted CCDs exhibit a mild flux dependence in the registration of charges. This is believed to be due to the accumulation of charges in the pixels altering the local electric field, effectively creating a repulsive force that scales linearly with the amount of charge already present (Antilogus et al. 2014) ${ }^{13}$ The most apparent consequence is a flux dependence of the PSF width, hence the effect being dubbed the 'brighter fatter relation'. The effect is not quite isotropic, having a preferred alignment towards the readout direction.

A proper correction of this effect would involve modelling the redistribution of charges and locally re-assigning image counts between neighbouring pixels to recreate the theoretical 'zero-flux' shape of stars and galaxies. Such an approach is currently under development, but goes beyond the scope of this paper. In the following sections, we will adopt a simpler approach, in which we eliminate the brightest stars when building the PSF model (see Appendix A for details). As they carry most of the photons, we need to compensate by pushing the star selection for the PSF model to fainter levels, where identification and shape measurement of stars can be performed much more reliably on co-added images.

\subsubsection{Star selection and PSFEx models}

The first step of building a PSF model is to select a sample of stars, from which the shape of the PSF can be reliably inferred. Because of the large size of DECam, we need to be able to tolerate considerable variations of size and ellipticity of actual stars in this initial selection to avoid forming an incomplete model of the PSF.

We work with the co-add catalogues from SExTRACTOR and perform a first-pass selection of stars in the size-magnitude plane (to be precise, in the plane of MAG_AUTO and both FWHM_IMAGE and FLUX_RADIUS), which yields mostly isolated stars, well suited for PSF measurements. To avoid saturated or noise-dominated stars, we could restrict the selection to objects with MAG_AUTO $\epsilon$ $[15,21.5]$, but the flux dependence of the PSF forces us to introduce a much more restrictive selection MAG_AUTO $\in[18,21.5]$ to prevent the brightest stars from rendering the PSF model inappropriate for the bulk of fainter stars and galaxies (cf. Fig. A1 for an example with the full range of stellar magnitudes).

We improve upon this first pass by requiring that stars be on the stellar branch in each of the filters $r, i, z$, which makes for a cleaner selection at faint levels and avoids the inclusion of blended stars whose faint companion is a drop-out galaxy for bluer filters. As a last step, we build a locally smoothed map of the FLUX_RADIUS measurements of the stars selected so far and reject $3 \sigma$ outliers. This localized selection is necessary for the wide-field imager DECam since stellar sizes increase considerably towards the edges of the field (cf. bottom left-hand panel of Fig. 3) so that mildly blended objects in the inner region could have passed the first selection with global size-magnitude cuts.

\footnotetext{
${ }^{13}$ We note that, in principle, the cosmic ray rejection in our image coaddition procedure (cf. Section 2.2) can introduce a similar effect (Heymans et al. 2012, their section 2.2). However, the change of the stellar width as a function of stellar flux is consistent with the one observed by Antilogus et al. (2014) on single-epoch images. We therefore conclude that our clipping procedure does not significantly affect the observed stellar widths.
} 

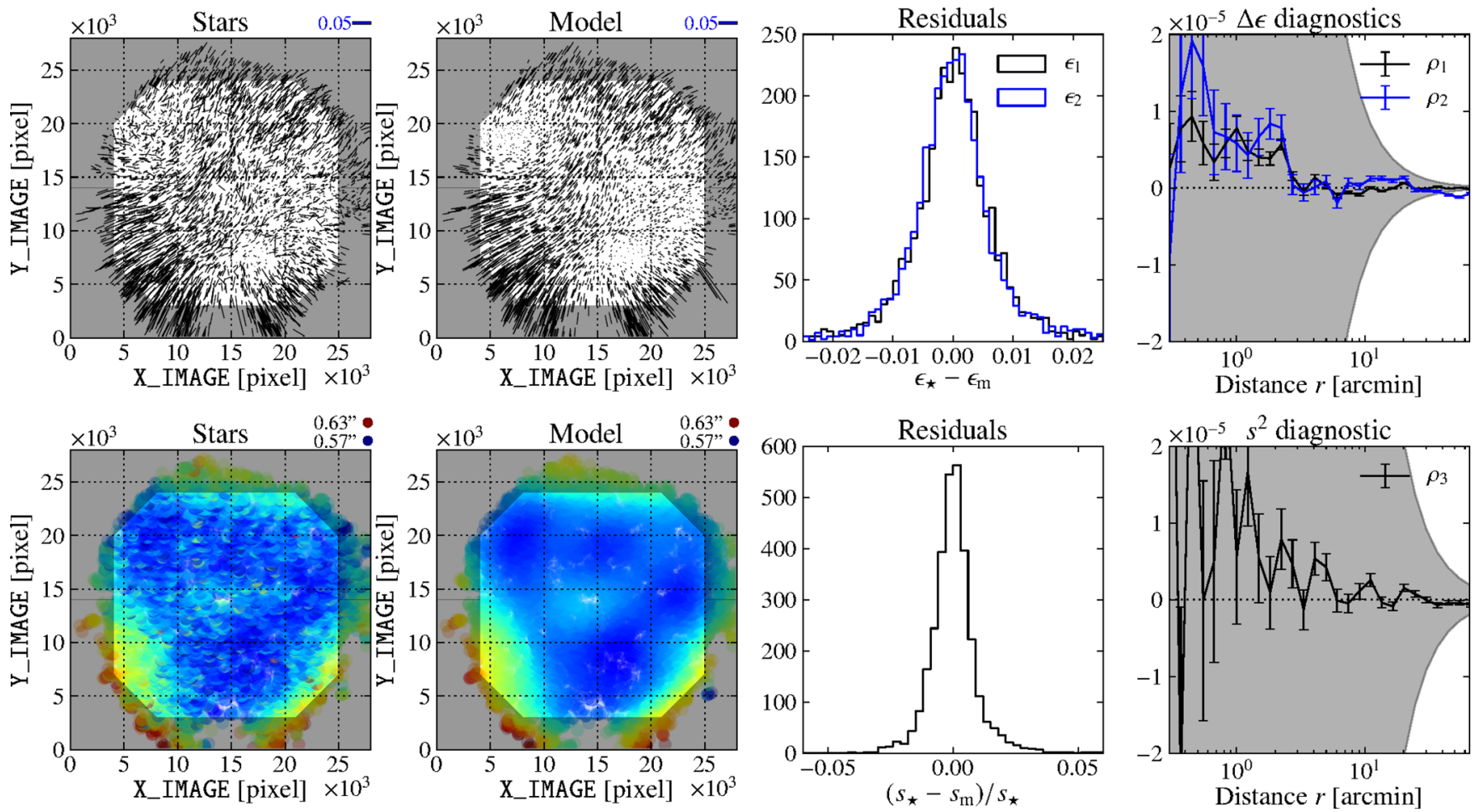

Figure 3. Stellar and PSF model ellipticities and sizes for the RXC J2248.7-4431 i.2 co-add image. From left to right, the top row shows the ellipticities $\epsilon_{\star}$ (in terms of shears, not polarizations, see e.g. equation 4.10 in Bartelmann \& Schneider 2001) of the stars from the clean catalogue (cf. Section 4.1.1); the corresponding ellipticities $\epsilon_{\mathrm{m}}$ of the PSFEX model derived from the same stars with PSFVAR_DEGREES=12; the residuals between stellar and model ellipticities; the diagnostic two-point correlation functions $\rho_{1}$ and $\rho_{2}$ out to a maximum separation of $1^{\circ}$. The bottom row is analogous but for stellar and PSF model sizes, respectively. See Section 4.1.2 for details on the diagnostic functions and their tolerances (equation 11 and shaded areas in the right-hand panels). In the two left-hand panels, the shaded area indicates the field cut to eliminate parts of the co-add images not covered by all exposures. For reference, a comparison whisker and the size colour scales are indicated in the top right-hand corner of these panels. The validation measurements are done with the moment-based shape code DEIMOS (Melchior et al. 2011), i.e. we determine the moments of both stellar images and pixelized PSFEx models at a fixed size of an adaptively matched elliptical Gaussian weight function of $\sigma_{\mathrm{w}}=2.5$ pixels $=0.658$ arcsec.

The procedure yields a very clean sample of objects, whose sizes are characteristic of relatively bright stars in the entire field with no noticeable contamination of neighbouring objects. This selection is then passed on as input catalogue to PSFEX (Bertin 2011), run with BASIS_TYPE=PIXEL_AUTO, BASIS_NUMBER $=20$ and varying polynomial degree PSFVAR_DEGREES $\in\{4,8,12,16\}$. The pixel-based model is therefore formed by polynomial interpolation of $20 \times 20$ superresolution cells, taken from $48 \times 48$ pixel cut-outs.

\subsubsection{PSF modelling tests}

Fig. 3 shows one example of our PSF modelling approach, displaying stellar and model ellipticities in the top row and corresponding sizes in the bottom row. We can see that both sizes and ellipticities tend to increase towards the field edges and that there are structures present at various scales in both measurements. We therefore assess the validity of the PSFEX models by the distribution of residuals and their cross-correlation functions, shown in the third and fourth column of Fig. 3, respectively.

Following Rowe (2010) (although using slightly different notation), we define the residual autocorrelation and the signal-residual cross-correlation functions of the (complex) ellipticity measurements:

$\rho_{1}(r) \equiv\left\langle\Delta \epsilon_{i}^{*} \Delta \epsilon_{j}\right\rangle_{i, j}$,

$\rho_{2}(r) \equiv\left\langle\epsilon_{i}^{*} \Delta \epsilon_{j}+\Delta \epsilon_{i}^{*} \epsilon_{j}\right\rangle_{i, j}$, where the average comprises pairs of stars $i, j$ with separations $r$, and the residuals are defined as $\Delta \epsilon \equiv \epsilon_{\star}-\epsilon_{\mathrm{m}}$, the difference of stellar and model ellipticities. Conjugation of the complex ellipticity is notated as $\epsilon^{*}$.

For the size measurements $s,{ }^{14}$ we introduce a third diagnostic function similar to the above,

$\rho_{3}(r) \equiv\left\langle\left(\frac{\Delta\left(s^{2}\right)}{s_{\star}^{2}}\right)_{i}\left(\frac{\Delta\left(s^{2}\right)}{s_{\star}^{2}}\right)_{j}\right\rangle_{i, j}$,

based upon the fractional error in $s^{2}$. These two sets of diagnostic functions check for the anisotropic and the isotropic validity of the PSF model and hence for the amount of systematic shear misestimation introduced by insufficient PSF correction.

After defining the diagnostic functions, we need to answer the question: how small do they need to be? In Appendix B, we work out the details, but the guiding principle is as follows. The error on the measured (deconvolved) shapes will be related to errors in the PSF model via a factor $T$ that compares the PSF size to the galaxy size. If we limit this PSF-induced shape measurement error by the intrinsic shape scatter of the background sample $\sigma_{\epsilon}$, which provides

\footnotetext{
${ }^{14}$ By which we mean the sum of the flux-normalized second-order moments $s^{2}=\frac{1}{F}\left(Q_{11}+Q_{22}\right)$, or equivalently the intensity-weighted second moment of the radius $\left\langle r^{2}\right\rangle_{I}$ averaged over image $I$.
} 
the fundamental limit to the statistical power of the lensing data, we can solve for the maximum tolerances of these diagnostics:

$\rho_{1}(r)+\left[\sigma_{\epsilon}^{2}+\left\langle\epsilon_{\star}^{*} \epsilon_{\star}\right\rangle(r)\right] \rho_{3}(r)<\frac{T^{2} \sigma_{\epsilon}^{2}}{n_{\text {gal }} \pi\left(R_{\max }^{2}-R_{\min }^{2}\right)}$,

where $T=P_{\gamma}\left(\frac{s_{\text {gal }}}{s_{\star}}\right)^{2}$, with $P_{\gamma}$ denoting the shear responsivity and $s_{\text {gal }}$ the size of the galaxy prior to convolution with the PSF, $n_{\text {gal }}$ is the number density of galaxies with shape measurements, for which we adopt $n_{\text {gal }}=10 \operatorname{arcmin}^{-2}$ per filter as typical value (cf. Section 4.3). ${ }^{15}$ The limits of the radial bin centred at $r$ are given by $R_{\max }$ and $R_{\min }$. As we point out in Appendix B, we drop the requirement on $\rho_{2}$ as it does not exhibit substantial diagnostic power.

Measuring these diagnostics and comparing them to the maximum tolerance then allow us to determine how large a typical galaxy has to be so that the PSF-induced errors do not dominate over the shape scatter. In units of IM3SHAPE's FWHM_RATIO (cf. Section 4.2 for the definition and equation B8 for the relation to $T$ ), our best models can use galaxies with FWHM_RATIO $\geq 1.1$. At our typical seeing of $\lesssim 1$ arcsec, this limit corresponds to $s_{\text {gal }} \approx 0.4$ arcsec, in line with measurements for the typical sizes of galaxies at our depth based on deep Hubble Space Telescope (HST) imaging in Miller et al. (2013, cf. their fig. 1). We do not consider PSF models, where that requirement would go beyond FWHM_RATIO $=1.2$ (or $s_{\text {gal }}$ $>0.65$ arcsec), the size cut, for which the shape measurement code is well tested (see next section). All fields and filters that qualify under this requirement are listed with a $\sqrt{ }$ in Table 2.

In terms of complexity, the best performing PSFEX models have polynomial degree between 8 and 16, with a majority at 16 . In addition and as replacement of the proposed role of $\rho_{2}$ in Rowe (2010), we performed a cross-validation study, in which we build a model using only a subset of the stars and then compute the diagnostic functions from the remaining stars. Even with the highest polynomial degrees, the models showed no indication of overfitting the data and, with the numbers of stars we provided, produced stable results in the analysed areas of each field.

To our knowledge, this is the first time that tolerances for PSF model diagnostics have been utilized to predict what size galaxies need exceed so that their shapes can be sensibly determined. The approach we have adopted here is conservative in three distinct ways. First, because we require the PSF models to stay within their tolerances at all scales, the actual PSF errors are smaller than our limits at most scales. Second, by calculating the tolerance at the limit of the smallest galaxies, the majority of the source sample will be less affected by PSF errors than predicted. And third, as we show in Section 4.3, the final shape catalogues are combined from different filters, so that the variation of PSF properties between filters enables a partial cancellation of the PSF-induced errors. We therefore consider the resulting shapes not to be dominated by PSF systematics, a claim we are going to review in Section 4.4.

\subsection{IM3SHAPE measurements}

For the weak lensing shear analysis presented in this paper, we use the publicly available galaxy shape measurement code IM 3 SHAPE $^{16}$

\footnotetext{
${ }^{15}$ In Section 4.3 we will introduce additional lensing weights that effectively reduce $n_{\text {gal }}$ by $10-15$ per cent, which means that our PSF diagnostics limits are slightly overconservative.

${ }^{16} \mathrm{https}: / /$ bitbucket.org/joezuntz/im3shape/; revision c8e6728.
}

(Zuntz et al. 2013). By maximizing the likelihood, it fits a PSFconvolved two-component bulge-plus-disc galaxy model to measure the ellipticity of each galaxy. In particular, we model galaxies as a sum of co-elliptical Sérsic (1963) profiles described by seven free parameters: ellipticity $\left(\epsilon_{1}, \epsilon_{2}\right)$, position $\left(x_{0}, y_{0}\right)$, disc half-light radius $\left(r_{\mathrm{d}}\right)$, bulge and disc peak flux $\left(A_{\mathrm{b}}, A_{\mathrm{d}}\right)$. We set the indices of the Sérsic profiles to 1 for the disc component and 4 for the bulge component. The bulge-disc size ratio is also kept fixed at 1.0.

To counter the adverse effects of aliasing and avoid upsampling biases we render intermediate model images at higher resolution as described in detail in Section 4.1 of Zuntz et al. (2013), choosing IM3SHAPE's upsampling parameters conservatively as UPSAMPLING $=5$, N_PIXELS_TO_UPSAMPLE $=8$, and N_CENTRAL_PIXEL_UPSAMPLING $=7$ with a postage stamp of size STAMP_SIZE $=37$ pixels. This is done for both the galaxy model image and the PSF image sampled from the PSFEX model at the galaxy position estimated with SExTRACTOR. The convolution of the two is then performed in Fourier space.

There are notable complications in our use of IM3SHAPE here compared to the simulation study presented by Zuntz et al. (2013).

Neighbouring objects. The flux of nearby objects affects shape measurement and leads to biases if not treated carefully. To this end, we make use of the segmentation map provided by SEXTRACTOR: we give zero weight to all pixels that are assigned to another identified object within the processed image stamp.

Background treatment. Although the single-filter co-add images are globally background-subtracted by SWARP, we find a non-zero local background for a few postage stamps where the global background subtraction remained insufficient. Therefore, we perform a local background estimation by averaging those pixels within the postage stamp that have not been assigned to any detection. The resulting value is then subtracted as a constant from all pixels within the analysed image.

We process each single-filter co-add image independently. For each image we run on all detected objects (with the exception of very bright and very faint objects). To clean the final shear catalogues, we first perform a star-galaxy separation based on the IM3SHAPE-estimated FWHM_RATIO between the pixel-PSF-convolved model image and the PSF image. The full width at half-maximum (FWHM) of a pixel-PSF-convolved galaxy model is estimated from its centred, noise-free model image with its ellipticity set to zero. The FWHM_RATIO is in effect a measure of the pre-seeing mean radius of the source, and hence we expect cuts based on this quantity to be free of first-order biases towards alignment with the PSF. All objects with FWHM_RATIO $<1.1$ are considered unreliable for shape measurement, often stars, and are excluded from further processing. We also remove shape measurement outliers by applying additional cuts based on IM3SHAPE fit results. In particular, we apply cuts for the following parameters: the best-fitting likelihood value, the minimum and maximum model value (per pixel), the minimum and maximum residual value (per pixel), the change in the estimated centroid position, and the disc half-light radius. The cuts are adjusted for each co-add image and are only meant to reject obvious failures of the shape measurement process.

\subsubsection{Noise-bias calibration}

Shape measurements are affected by a prominent bias when the galaxy images become noisy (e.g. Massey et al. 2007). This is a consequence of the observable, the galaxy ellipticity, being 


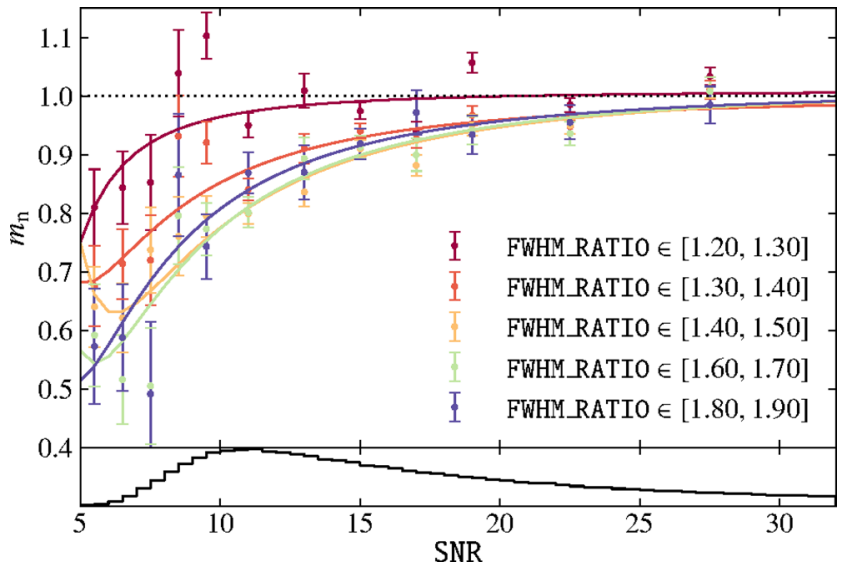

Figure 4. Noise bias on the multiplicative term $m_{\mathrm{n}}$ of the shear response as a function of IM3SHAPE'S SNR for different values of the galaxy FWHM_RATIO. The solid lines are even-order polynomial fits to the data (cf. equation 13). The bottom panel shows the SNR distribution of galaxies with shape measurement from any single-filter co-add image, averaged over all fields and riz filters.

non-linearly related to the flux in each pixel and applies to modelfitting methods and moment-based measures of the ellipticity alike (Kacprzak et al. 2012; Melchior \& Viola 2012; Refregier et al. 2012).

To calibrate IM3SHAPE's response to noise bias we simulate mock galaxies, using the GALSIM ${ }^{17}$ (Rowe et al. 2014) framework. In particular, we adopt the methodology of Mandelbaum et al. (2012) and degrade high-resolution and high-significance images from Cosmic Evolution Survey (COSMOS) to the DECam resolution and magnitude limit (cf. Fig. 1). We approximate the co-add PSF by a circular Moffat (1969) profile with seeing values $\in[0.7,0.8,0.9]$ arcsec, spanning the expected range of our observing conditions. Applying exactly the same cuts as for shape catalogues from the co-add images, we have verified that both magnitude and size distributions of the simulated galaxies closely match the observed ones. Adding an artificial shear $\gamma$ of order 5 per cent, we can infer the shear response,

$m_{\mathrm{n}} \equiv \frac{\partial\langle\epsilon\rangle}{\partial \gamma}$,

as a function of the signal-to-noise ratio SNR and FWHM_RATIO. We believe these two parameters to largely determine the shear response, and by working with size ratios we render this calibration mostly insensitive to the observed PSF widths that occasionally exceeded the simulated range. The result is shown in Fig. 4. At high SNR, the shear can be measured in an unbiased fashion for all galaxy sizes, whereas the noise bias gets progressively worse for lower SNR, scaling roughly as SNR ${ }^{-2}$, consistent with findings of Bernstein \& Jarvis (2002). It is counter-intuitive that the smallest galaxies show the least amount of bias. Also, at very low SNR the larger galaxies show an intriguing upturn. We interpret both as higher order effects of the noise bias. A decrease in noise bias for smaller galaxies is plausible, particularly for a mixture of biases with different signs at different orders in SNR and FWHM_RATIO, with perhaps some fortuitous cancellation for the smallest galaxies we plot. According to Kacprzak et al. (2012), only even orders of SNR can appear in the noise-bias

\footnotetext{
${ }^{17}$ https://github.com/GalSim-developers/GalSim
}

Table 3. Best-fitting parameters of equation (13) to the simulated data from Fig. 4. The last column indicates the percentage of all galaxies with shape measurements in any band to fall into the given bin, averaged over all fields and riz filters. The missing fraction of about 7 per cent are larger than those listed here but show an identical noise bias, we therefore adopt the FWHM_RATIO $=1.9$ fit to correct them.

\begin{tabular}{lcrrc}
\hline FWHM_RATIO & $c_{0}$ & \multicolumn{1}{c}{$c_{2}$} & \multicolumn{1}{c}{$c_{4}$} & $\begin{array}{c}N_{\text {gal }} \\
\text { (per cent) }\end{array}$ \\
\hline$\in[1.20,1.30]$ & $1.010 \pm 0.012$ & $-3.9 \pm 3.6$ & $-64 \pm 129$ & 24.2 \\
$\in[1.30,1.40]$ & $1.001 \pm 0.012$ & $-17.2 \pm 3.4$ & $229 \pm 127$ & 19.0 \\
$\in[1.40,1.50]$ & $1.019 \pm 0.013$ & $-30.2 \pm 3.5$ & $587 \pm 130$ & 15.3 \\
$\in[1.50,1.60]$ & $1.028 \pm 0.016$ & $-30.5 \pm 4.1$ & $498 \pm 150$ & 12.0 \\
$\in[1.60,1.70]$ & $1.019 \pm 0.018$ & $-28.9 \pm 4.8$ & $438 \pm 174$ & 9.8 \\
$\in[1.70,1.80]$ & $1.042 \pm 0.021$ & $-28.3 \pm 5.4$ & $326 \pm 193$ & 7.3 \\
$\in[1.80,1.90]$ & $1.014 \pm 0.024$ & $-23.4 \pm 6.2$ & $271 \pm 214$ & 5.0 \\
\hline
\end{tabular}

relation, therefore, we attempt to parametrize the dependence with the following polynomial model,

$m_{\mathrm{n}} \approx c_{0}+c_{2} \mathrm{SNR}^{-2}+c_{4} \mathrm{SNR}^{-4}$,

whose best-fitting parameters are listed in Table 3. The applied noise-bias corrections are taken from these fits in each of the size bins.

We also note that the shear response of equation (12) is assumed to be linear in the shear. Large shears, as encountered close to the centres of clusters, may introduce a non-linear response, which would be overseen by our calibration approach. However, for the analysis in Section 5 we will exclude these inner regions and restrict ourselves to shear values, for which IM3SHAPE's performance was tested by our simulations and others (e.g. Kitching et al. 2012).

\subsection{Final catalogue creation}

For each cluster field we include the shape catalogues for all filters $f$ that passed the PSF modelling requirements from Section 4.1.2 (cf. Table 2 for the list). Based on SExTRACTOR measurements, we enforce additional cuts to clean the catalogues from potentially problematic measurements: FLAGS $=0$ and CLASS_STAR $<0.8$. We furthermore exclude areas at the edges of the fields, where the coverage is not homogeneous across filters, giving rise to the 'picture frame' geometry shown in the left-hand panels of Fig. 3. We also mask out stars detected in the Tycho-2 catalogue (Høg et al. 2000) with magnitude-dependent circular and hand-crafted bleed trail masks to avoid saturation features and local sky-background variations that could affect the photometry or the shape measurements. The remaining sample is shown as 'Clean' in Fig. 1.

On top of the selection inherent to IM3SHAPE success, we restrict the shape measurements to SNR $>5$ and FWHM_RATIO $>1.2$ in concordance with limits on both the PSFEX-model quality and the shape calibrations in Section 4.2.1. For each remaining galaxy $j$, the shapes are combined from all available filters $f(j)$ according to their IM3SHAPE weight $w_{3}(j, f)$,

$\epsilon(j)=\frac{\sum_{f(j)} m_{\mathrm{n}}(j, f)^{-1} \epsilon(j, f) w_{3}(j, f)}{\sum_{f(j)} w_{3}(j, f)}$.

This filter-combined ellipticity maximizes the number of galaxies with shape measurements and reduces the variance from pixel noise in each ellipticity estimate derived from more than a single filter. The resulting catalogue forms the basis for all subsequent analysis and is denoted as the 'Shapes' sample in Fig. 1. The number density $n_{\text {gal }}$ of this catalogue ranges from 9 to $12 \operatorname{arcmin}^{-2}$, with the best 
available seeing in each field being the dominant factor of that variation. This is consistent with the expectations for the full depth DES imaging data (The Dark Energy Survey Collaboration 2005). Only the co-add images of the additional cluster Abell 3261 do not reach the nominal full depth of 10 exposures, resulting in a reduced $n_{\text {gal }}=8 \operatorname{arcmin}^{-2}$.

Note that the noise-bias correction $m_{n}(j, f)^{-1}$ in equation (14) depends on SNR $(j, f)$ and FWHM_RATIO $(j, f)$ as described in Section 4.2.1. The weight takes both statistical and measurement variances into account (e.g. Hoekstra, Franx \& Kuijken 2000, their equation A2):

$w_{3}(j, f)=\frac{\sigma_{\epsilon}^{2}}{\sigma_{\epsilon}^{2}+\left[0.1 \frac{1}{m_{\mathrm{n}}(j, f)} \frac{20}{\operatorname{SNR}(j, f)}\right]^{2}}$,

where we have adopted an estimator for the measurement error $\sigma_{j}$ that scales inversely with SNR and accounts for variable amounts of noise-bias correction. This estimate may be on the conservative side, but has performed well in simulations, where a full likelihood exploration for the parameters was available. For the weight $w_{3}(j)$ of the combined shapes, we stick to the formula above, but replace $\operatorname{SNR}(j, f)$ with $\sum_{f(j)} \operatorname{SNR}(j, f)$.

When we apply the weights to the shape catalogue, the effective number density $n_{\text {eff }} \approx 0.87 n_{\text {gal }}$, while both shape scatter and mean redshift of all galaxies with shape measurements are only mildly (i.e. of order 5 per cent) reduced by the weighting, $\left\langle z_{\text {phot }}\right\rangle \approx 0.7$ and $\sigma_{\mathrm{e}} \approx 0.3$. We emphasize that because of anticipated changes in the shear measurement pipeline for future studies, these numbers are only roughly indicative of the DES performance and should therefore be treated with due caution.

Applying the photo- $z$ cut of equation (3) finally yields the shape catalogue for the background sample, labelled as 'BG' in Fig. 1.

\subsection{Consistency tests}

While not strictly necessary for the lensing analysis in Section 5, we choose to express the measurements in $\Delta \Sigma(r)$ rather than in the actual observable, the shear $g=\langle\epsilon\rangle$. This physical quantity should be invariant under choice of source populations, at least ideally. It allows us to slice the background sample in various ways and thereby to test whether the shear measurements and the various calibrations are accurate. To work with a sufficient number of galaxies, we combine the four clusters by stacking them at their respective (BCG) centres. The test results are shown in Fig. 5.
First, we check whether the typical SNR dependence of weak lensing measurements is corrected by our calibrations from Section 4.2.1. The left-hand panel of Fig. 5 shows that there is no strong trend visible when varying IM3SHAPE's SNR parameter, implying that our calibration was indeed successful. In terms of weak lensing mass (see methodology in Section 5.1), we have less than 10 per cent variations between each subset and the entire stack. Note that we slice the final catalogue, where ellipticity measurements have been combined according to equation (14), therefore, the SNR is given as the sum over the measurements in each filter. The pivotal value $\sum_{f}$ SNR $=30$ is chosen here because it yields $\operatorname{SNR}(f) \approx 10$, which, according to Fig. 4, separates galaxies with substantial levels of noise-bias corrections from those with a much milder correction.

Second, we split the sample according to the reported photo- $z$ of each source. As we have corrected for the redshift dependence of the measured shear by converting to $\Delta \Sigma$ (see Section 3.4 for the details and calibrations we applied to the raw photo- $z$ values), we should not see any variation induced by the change in distances in equation (1), and indeed there is none recognizable in the second panel of Fig. 5. Quantitatively, we find the upper redshift slice to have a higher mass estimate of about 15 per cent, whereas the lower is about 10 per cent low in mass compared to the entire stack, neither of which is significant given the errors in the stacked lensing profiles. Note that this outcome is not trivial as both the photo- $z$ corrections and the noise-bias calibrations have to perform well. Because of the correlation between flux and distance, correcting only one of them is not sufficient to null this test.

Third, we revisit our claim from Section 4.1.2 that the PSFEX models allow us to use galaxies down to FWHM_RATIO $=1.2$ or even below. The third panel of Fig. 5 shows that this is unfortunately not entirely the case, with a mild size dependence of the reported $\Delta \Sigma$ : the larger (smaller) set of galaxies yields a mass estimate that is about 30 per cent higher (10 per cent lower) than the entire stack. While at rather low statistical significance - the errors on the mass estimate of each slice are of order 35 per cent - we suspect that correlated noise and complex PSF shapes in the co-add images are more harmful to small galaxies than indicated by the noisebias simulations from Section 4.2.1 that used uncorrelated pixel noise and simplistic PSFs. We want to point out that this tendency is barely recognizable in a stack of four clusters, so that we do not expect it to limit the individual lensing analyses in the next sections.

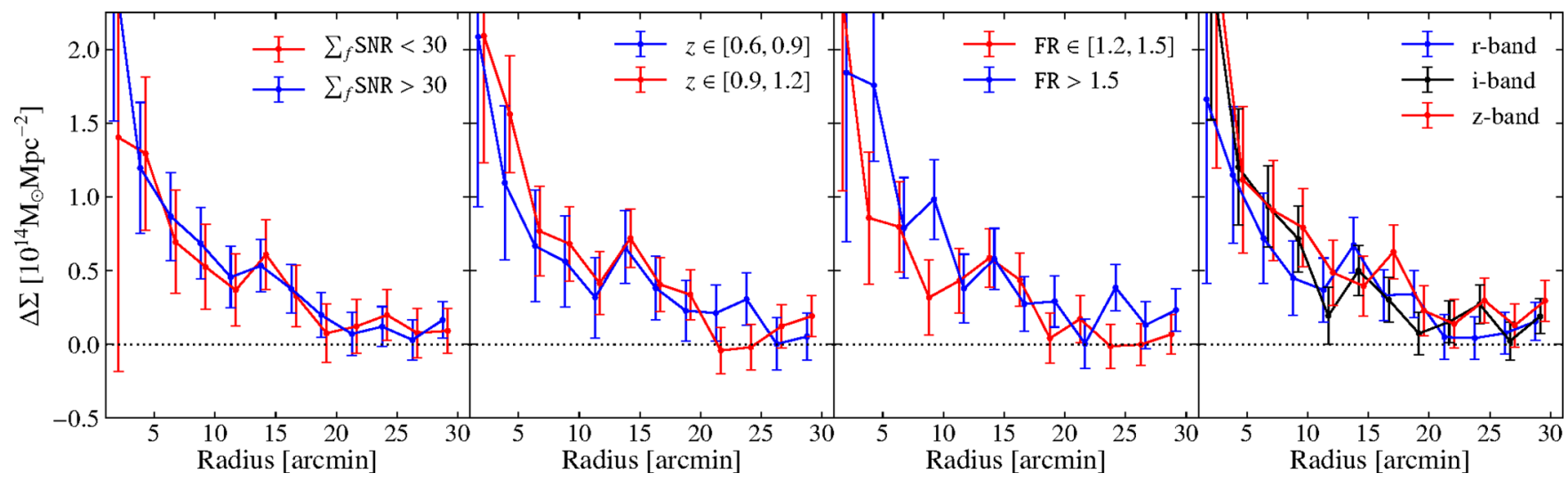

Figure 5. Consistency test for the background-selected shape catalogues, sliced in IM3SHAPE's $\sum_{f}$ SNR (first panel), in photo- $z$ estimate (second panel), in FWHM_RATIO (FR for short, third panel), and from different filters (fourth panel). The conversion between shear estimate and $\Delta \Sigma$ is done with $\Sigma_{\text {crit }}(z)$ calculated for each galaxy individually based on its photometric redshift according to equation (8). The numbers are stacked over the four fields, errors correspond to the dispersion of the weighted mean in each bin. For clarity, the points of different slices have been shifted horizontally by $0.4 \operatorname{arcmin}$. 
Finally, the last panel of Fig. 5 shows the lensing signal if we only use the shape measurements from single filters instead of combining them according to equation (14). Using single filters constitutes a drop-out technique, where galaxies are more likely measurable in redder filters if they are at higher redshifts. Since the redshift dependence of the signal seems to be well characterized (second panel), we expect consistent measurements here, too. However, uncorrected effects related to the CCD (e.g. prominent fringing in the $z$-band) or the instrument in general could interfere, but to the limit of this test we can rule this out: the mass estimates agree to better than 10 per cent across filters. This leads us to the non-trivial conclusion that DECam images taken in each of the riz filters seem equally suitable for shape measurements.

Note that this methodology effectively constitutes a sequence of null tests, even though we inspect the actual signal. We could have subtracted the mean signal to render it a proper null test, but we choose to leave it in since some of the systematics could scale with, for example, the lensing strength or the source density, so it may help to actually see the mean cluster signal to gauge the dependency on clustercentric distance $r$. Larger cluster samples investigated in forthcoming DES analyses will substantially sharpen these consistency tests and allow us to detect potential shape measurement problems with much higher precision.

\section{SHEAR PROFILES AND LENSING MASSES}

We now measure the lensing masses by fitting a radial profile to the tangential shear signal, centred on the BCG coordinates as listed in Table 1.

\subsection{NFW profile fits and lensing masses}

To obtain estimates of cluster masses, we assume the density profile described first by Navarro, Frenk \& White (1996). The threedimensional density $\rho(r)$ of the NFW profile at radius $r$ is given as

$\rho(r)=\frac{\rho_{0}}{\left(r / r_{\mathrm{s}}\right)\left(1+r / r_{\mathrm{s}}\right)^{2}}$.

The profile can alternatively be expressed in terms of the mass $M_{200 \mathrm{c}}$ and concentration $c_{200 \mathrm{c}}=r_{200 \mathrm{c}} / r_{\mathrm{s}}$, instead of the central density $\rho_{0}$ and scale radius $r_{\mathrm{s}}$. Here $r_{200 \mathrm{c}}$ denotes the radius of a sphere that comprises an overdensity of 200 times the critical density at the redshift of the cluster. The projected density and gravitational shear of the NFW profile are given in Bartelmann (1996) and Wright \& Brainerd (2000).

Assuming Gaussian errors on the shape estimates, ${ }^{18}$ the likelihood of any model can be calculated from the shear catalogue by means of the $\chi^{2}$ statistic. Given model predictions $\Delta \hat{\Sigma}(r)$ for the lens and the measurements of component $\epsilon_{\mathrm{t}}(j)$ of the ellipticity of galaxy $j$ tangential to the cluster centre, the likelihood $\mathcal{L}$ can be written as

$\ln \mathcal{L}=-\frac{1}{2} \sum_{j} \frac{\left[\Delta \hat{\Sigma}\left(r_{k}\right)-\Sigma_{\text {crit }}(j) \epsilon_{\mathrm{t}}(j)\right]^{2}}{\Sigma_{\text {crit }}^{2}(j)\left[\sigma_{j}^{2}+\sigma_{\epsilon}^{2}\right]}+$ const

where we use the corrected $\Sigma_{\text {crit }}$ from equation (8) and insert $w_{3}(j) / \sigma_{\epsilon}^{2}$ from equation (15) as the total variance term in the denom-

\footnotetext{
${ }^{18}$ That is an overly simplified assumption because measurement errors will induce Cauchy-like wings even if the intrinsic shape dispersion were Gaussian (Melchior \& Viola 2012; Applegate et al. 2014).
}

inator. Since the reduced shear in the weak lensing regime is a small correction to the intrinsic shape, the latter can still employ $\sigma_{\epsilon}$ from the observed (as opposed to an unlensed) distribution of shapes. We evaluate the posterior distribution of the likelihood $\mathcal{L}$ with the MCMC sampler EMCEE ${ }^{19}$ (Foreman-Mackey et al. 2013), adopting a log-normal prior on the concentration following the concentrationmass relation of Duffy et al. (2008, their 'full' sample) with scatter of $\sigma_{\log c}=0.18$ (Bullock et al. 2001).

To avoid the regions where the cluster-member contamination correction (cf. Section 3.4.1) and possible shape measurement errors due to crowding may not be well characterized, we exclude the region in the very centre and start the fit at $r=3 \mathrm{arcmin}$. This also renders us robust against miscentring as our choice of the BCG centre may not correspond to the actual gravitational centre of the halo. To limit the inclusion of uncorrelated large-scale structure or clusters, we also restrict the outer limit to $r=15 \operatorname{arcmin}$. The resulting range $3 \leq r \leq 15$ arcmin is similar to $750 \mathrm{kpc} \leq r \leq 3 \mathrm{Mpc}$ employed by Applegate et al. (2014) but extends somewhat farther out to reduce the shot noise from the rather low $n_{\text {gal }}$ of our data. The NFW profile is not a good fit to lensing measurements at such large distances because it lacks the two-halo contribution from structures associated with the clusters. However, the resulting bias is only of order 10 per cent (Oguri \& Hamana 2011, their fig. 4), which will certainly be below our measurement accuracy.

In Fig. 6 we show the individual shear profiles and 100 randomly chosen sample projections on to the data to demonstrate the range of viable models after an initial burn-in phase. Parameter confidences are given in terms of the 16th, 50th, and 84th percentiles of the marginalized mass $M_{200 c}$ and concentration $c_{200 c}$ distributions. We can see that SPTW1 is well fit by an NFW model, including the innermost radial bin that was not included in the fit. Given our uncertainties, the NFW profile constitutes an acceptable model for all clusters. We also want to point out that the B-mode, denoted as $\gamma_{\times}$in Fig. 6, is statistically consistent with zero for all clusters, although some moderately large fluctuations occur.

It is typical for pure weak lensing measurements that the concentration is only poorly constrained (e.g. Postman et al. 2012), a tendency that we have even exacerbated by excluding the inner 3 arcmin. This highlights the potential importance of a concentration prior, a situation in which the significant differences in the literature between concentration-mass relations derived from different simulations or observational studies may appear worrisome. However, deviations of the assumed relation from the truth only mildly impact the weak lensing mass measurement (e.g. Hoekstra et al. 2012, their section 4.3). Indeed, we find no significant differences of the marginalized results using the Duffy prior or an entirely flat prior within $0<c_{200 \mathrm{c}}<8$.

Comparing our $M_{200 c}$ estimates with previous results listed in Table 4, often based on substantially deeper data, we find good agreement for RXC J2248.7-4431, where the mass estimate in Gruen et al. (2013) is within our 68 per cent confidence region. Two recent analyses of the same data - together with magnification (Umetsu et al. 2014) or HST strong- and weak lensing constraints (Merten et al. 2014) - yield reduced estimates of $M_{200 c}$, which are fully consistent with our result.

For the Bullet cluster, our mass estimate is rather poor due to a fairly low $n_{\text {gal }}$, but we can recover the result of Clowe, Gonzalez \& Markevitch (2004) within errors. This comparison is, however, not as straightforward as it seems. The original ground-based Very

\footnotetext{
${ }^{19} \mathrm{http} / / /$ dan.iel.fm/emcee/
} 


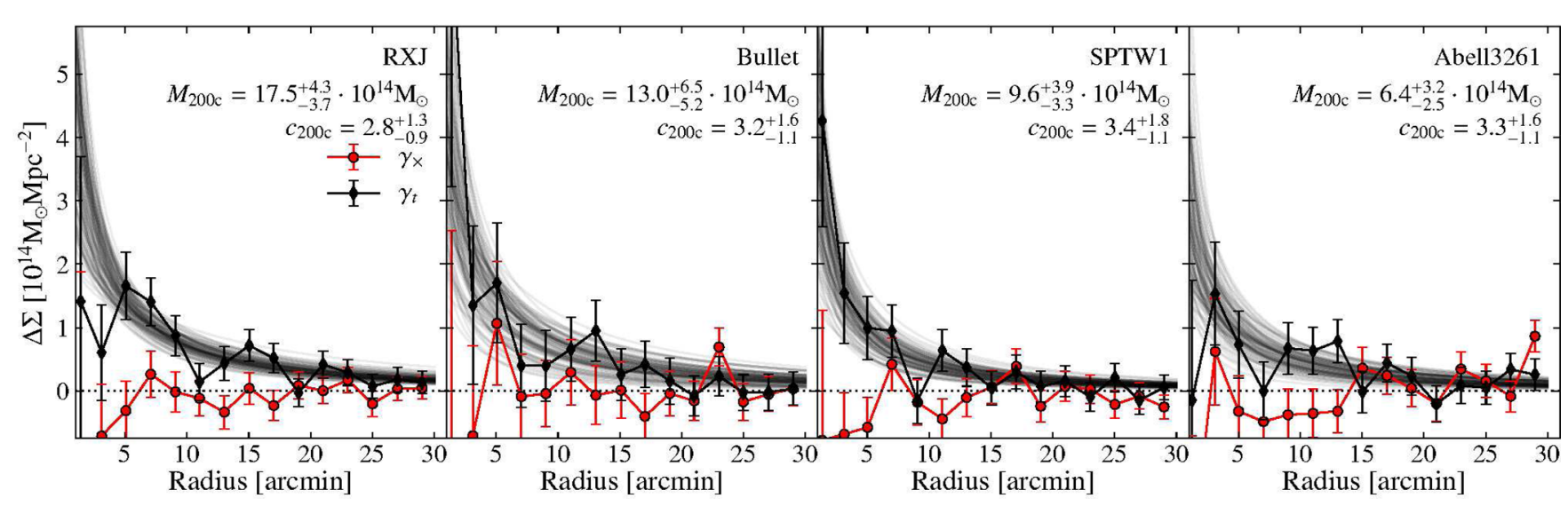

Figure 6. Surface density contrast $\Delta \Sigma=\Sigma_{\text {crit }} \gamma_{\mathrm{t}}$ profiles (black) for each of the four cluster fields and 100 random MCMC sample projections on to the data (light grey) after an initial burn-in phase. The fit range was restricted to $3 \leq r \leq 15$ arcmin. The B-mode $\Sigma_{\text {crit }} \gamma \times$ is shown in red.

Table 4. Weak lensing masses $M_{200 c}$ in units of $10^{14} \mathrm{M}_{\odot}$ (with a log-normal prior on $c_{200 c}$ based on the Duffy et al. 2008 concentration-mass relation), redMaPPer richness $\lambda$ and redshift estimate $z_{\lambda}$, and their statistical errors (see Sections 3.2 and 5.1 for details). The literature mass estimates are derived from weak lensing, galaxy dynamics (D), or optical richness (R).

\begin{tabular}{|c|c|c|c|c|}
\hline Cluster name & $M_{200 \mathrm{c}}$ & $\lambda$ & $z_{\lambda}$ & Literature value $M_{200 \mathrm{c}}$ \\
\hline SCSO J233227-535827 & $9.6_{-3.3}^{+3.9}$ & $77 \pm 4$ & $0.391 \pm 0.008$ & $11.2_{-2.7}^{+3.0}$ (Gruen et al. 2014b), $4.9 \pm 3.3 \pm 1.4$ (High et al. 2010, R) \\
\hline Abell 3261 & $6.4_{-25}^{+3.2}$ & $71 \pm 3$ & $0.216 \pm 0.003$ & - \\
\hline
\end{tabular}

Notes. ${ }^{a}$ We converted the measured $r_{200 \mathrm{c}}$ from Clowe et al. (2004), which lacks an error estimate, to $M_{200 \mathrm{c}}$ using the critical density in our adopted cosmology.

Large Telescope (VLT) data in Clowe et al. (2004) had a field of view of only $7 \mathrm{arcmin}$, hence the radial range probed there is almost entirely excluded in our fit that starts at 3 arcmin. We therefore acknowledge the similarity of our mass estimates with the literature value, but do not consider this a powerful result.

The situation is different for SCSO J233227-535827, where the shear profile is more regular and our mass estimate is better constrained. Our estimate is in excellent agreement with the weak lensing analysis from Gruen et al. (2014b). Our central value is about twice as high as the estimate from High et al. (2010) based on optical richness. Another recent mass estimate from Sunyaev-Zel'dovich (SZ) and X-ray scaling relations by Reichardt et al. (2013) of $M_{500 \mathrm{c}}=6.50 \pm 0.79 h_{70}^{-1} 10^{14} \mathrm{M}_{\odot}$ is again fully consistent with our lensing estimate, which we derive as $M_{500 \mathrm{c}}=6.4_{-2.2}^{+2.6} \times 10^{14} \mathrm{M}_{\odot}$ by assuming an NFW profile with $c_{200 c}=3.4$ as measured from our lensing data.

We conclude this section with a test on the robustness of the mass estimate against uncertainties in the numerous calibrations we have employed. To assess the impact of the calibrations, we repeated the NFW profile fitting without the calibrations. The cluster-member contamination correction from Section 3.4.1 alone increases the mass estimates by less than 5 per cent as it only affects the galaxies within $\approx 5$ arcmin, and our fits start at 3 arcmin. The photo- $z$ recalibration from Section 3.4.2 yields a global boost of the lensing signal by $5-10$ per cent. The biggest impact stems from the noise-bias correction (Section 4.2.1), which globally increases the inferred shear by $\approx 20$ per cent. ${ }^{20}$ The sum of all these calibrations amounts to a

${ }^{20}$ This amount of noise-bias correction seems high by cosmic shear standards, but we also include much fainter galaxies with our adopted cuts. considerable 35 per cent, so that uncertainties in the calibrations actually become important. As we have laid out in the relevant sections, these calibrations are determined quite well with dedicated measurements, but we will conservatively allow for a 20 per cent systematic error budget. Compared to the statistical uncertainties that are of order 50 per cent (with RXC J2248.7-4431 being the only cluster with a 25 per cent statistical error), we conclude that the overall error is dominated by shape noise from the dispersion of galaxy ellipticities.

\subsection{Richness-mass relation}

An obvious additional cross-check for the data in this work is to compare it with the mass-richness relation for low-redshift clusters. Rykoff et al. (2012) constrained it with maxBCG (Koester et al. 2007) clusters in the range $0.1 \leq z \leq 0.3$ with a very similar richness estimator $\lambda$ to the one we employ here. Although their redshift range only covers two of our clusters (the other two are at slightly higher redshift), we expect that deviations would more likely stem from our large measurement errors on the weak lensing mass than from any possible redshift evolution of that relation.

We list the redMaPPer-estimated richness and redshift estimates in Table 4 and note that for the three clusters, for which we have spectroscopic redshifts, redMaPPer provides excellent redshift estimates, with deviations within $2 \Delta z_{\lambda}$ in all cases. We take this as an indication that our overall photometric calibration (Section 3.1) and the red-sequence (Section 3.2) calibration were successful. In

Similar levels of noise bias have been reported for various methods e.g. by Massey et al. (2007, their section 5.5). 


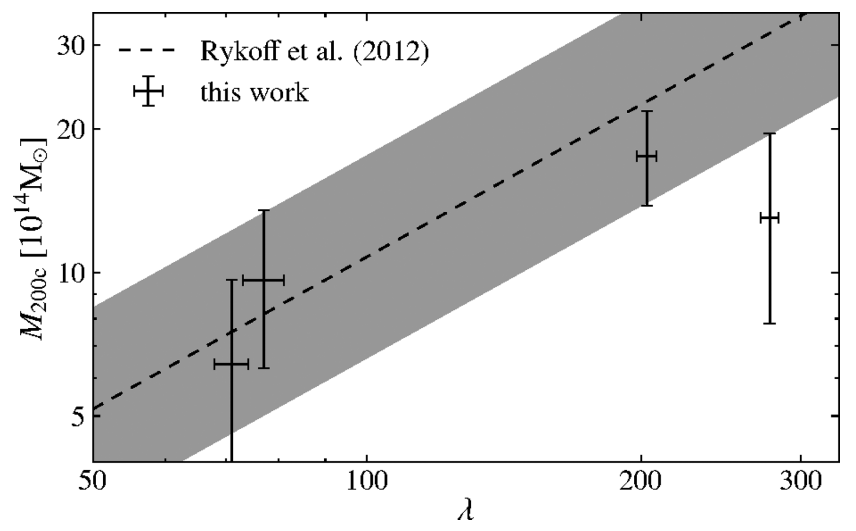

Figure 7. Lensing mass $M_{200 \mathrm{c}}$ as a function of redMaPPer's richness $\lambda$ for the four investigated clusters. The dashed line shows the expected scaling relation from Rykoff et al. (2012, see equation 18) with their proposed relative scatter of 33 per cent at fixed richness (shaded region).

Fig. 7 we compare the richnesses with the weak lensing masses from Fig. 6 and the best-fitting solution from Rykoff et al. (2012, their equation B5),

$\ln \left(\frac{M_{200 c}}{10^{14} h^{-1} \mathrm{M}_{\odot}}\right)=1.48(1 \pm 0.33)+1.06 \ln \left(\frac{\lambda}{60}\right)$,

and find that our measurements indeed agree with the expectations ${ }^{21}$ within the considerable scatter both measurements exhibit.

\section{MASS AND CLUSTER GALAXY DISTRIBUTIONS}

We now move from spherically averaged masses to two-dimensional maps of the weak lensing mass and the cluster galaxies. We have seen in Section 5.1 that the NFW profile is an acceptable fit to the measurements. In detail, that is not even expected as the NFW profile only describes the average radial profile of dark matter haloes in simulations, incapable of reproducing the complex structures massive clusters often exhibit (e.g. Merten et al. 2011; Medezinski et al. 2013). We are particularly interested in the environment of these clusters, using DECam to follow the filamentary structures from which the clusters accrete out to distances normally not accessible to dedicated cluster-lensing studies on imagers with smaller fields of view.

We start the inspection of the cluster fields visually at the central $5 \times 5 \operatorname{arcmin}^{2}$ of each cluster in the left-hand column of Fig. 8, where we can see the BCG and other bright cluster members. In all four clusters, we can see that several of the obvious cluster member galaxies tend to line up along one axis that coincides with the orientation of the BCG. This long-known tendency (Sastry 1968; Carter \& Metcalfe 1980) is still not entirely understood, but a plausible scenario entails that accretion of satellite haloes along filaments determines the cluster major axis, and the BCG orients itself accordingly (e.g. Hao et al. 2011, and references therein).

\footnotetext{
${ }^{21}$ We note that Rykoff et al. (2012) made simplifying assumptions that entail e.g. the absence of an error on the slope in equation (18). We therefore refer to their appendix B for a discussion of the limitation of the inferred mass-richness relation.
}

\subsection{Mass maps}

To perform the mass reconstruction of the galaxy clusters, we move further out to cover 30 arcmin, a scale typical of weak lensing studies of individual galaxy clusters, and employ the aperture-mass technique from Schneider (1996). It exploits that a local estimate of the convergence $\kappa(\theta)=\Sigma(\theta) / \Sigma_{\text {crit }}$ can be obtained by summing up all ellipticity measurements $\epsilon_{\mathrm{t}}\left(\theta_{j}\right)$ inside of a circular aperture,

$M_{\mathrm{ap}}(\theta)=\sum_{j} Q\left(\left|\theta-\theta_{j}\right|\right) \epsilon_{\mathrm{t}}\left(\theta_{j}\right)$.

Here the tangential component of the ellipticity $\epsilon_{\mathrm{t}}$ is calculated with respect to the centre $\theta$ of the aperture, not the centre of the cluster as in equation (17). Under the assumption of uncorrelated Gaussian noise in the ellipticities with variance $\sigma_{\epsilon}^{2}$, the variance of $M_{\text {ap }}$ is given by

$\sigma_{M_{\mathrm{ap}}}^{2}=\frac{1}{2} \sum_{j} Q^{2}\left(\left|\theta-\theta_{j}\right|\right)|\epsilon|^{2}\left(\theta_{j}\right)$.

So far we have not specified the weight function $Q$, and in fact we have considerable freedom in doing so, which allows us to demand additional desirable properties of the reconstructed mass maps. Since the noise contribution stemming from $\sigma_{\epsilon}$ is scale-free, the maximum $M_{\text {ap }} / \sigma_{M_{\text {ap }}}$ is achieved if $Q$ is identical to the signal we try to find, i.e. the tangential shear of the cluster (Schneider 1996; Bartelmann \& Schneider 2001). Thus, we could turn the measured shear profiles from Fig. 6 into templates for optimal individual shapes of $Q$, which would result in mass maps that are not easily comparable against each other. We therefore seek a common weight function shape $Q(r)$ with a single characteristic radius $R_{\text {ap }}$, knowing that we will sacrifice some statistical significance with this decision. We follow the design choices of Schneider (1996), which we find particularly suitable for this work for three reasons. First, he approximated the shear profile by an isothermal $\gamma_{\mathrm{t}} \propto 1 / r$ relation, which should allow us to capture the extended environment of these massive, and in parts even merging, clusters better than the steeper NFW profile. Second, the weight function excises an inner circle at $r<v_{1} R_{\text {ap }}$ to avoid regions where the relation between $\gamma$ and $\kappa$ becomes non-linear and shape measurements are rendered difficult due to bright cluster members. The same concern has led us to exclude the inner regions when fitting the NFW profile in Section 5.1. Third, Schneider (1996) also sets the outer edge of the weight function at $r>R_{\text {ap }}$ to allow the filter to operate on finite, and potentially masked, fields and to avoid the inclusion of truly uncorrelated structures, again corresponding to decisions we made earlier. To avoid a sharp cut-off at that outer edge, we let the filter roll off smoothly, starting at $v_{2} R_{\text {ap }}$, where $v_{2}<1$. Considering the scale, over which we can find a noticeable shear signal in Fig. 6, we choose $R_{\text {ap }}=10 \mathrm{arcmin}$, with an inner exclusion region of $v_{1} R_{\text {ap }}=1$ arcmin and the onset of the roll-off at $v_{2} R_{\text {ap }}=9$ arcmin. The exact form of the $Q(r)$ can be seen in Schneider (1996, equation 34, figs 1 and 2). It is worth pointing out that in adopting these choices we employ a filter that is substantially different from those that attempt to maximize purity of blind detections from wide-field weak lensing data (e.g. Maturi et al. 2005) by suppressing the influence of large-scale structure fluctuations: here we know where the clusters are and we want to probe the correlated material surrounding the clusters.

We present the resulting mass maps, i.e. maps of $M_{\mathrm{ap}} / \sigma_{M_{\mathrm{ap}}}$ centred on the location of the BCG, as contours in the middle panels of Fig. 8. The mass maps of RXJ and SPTW1 show clearly significant peaks, exceeding $5.5 \sigma$ and $3.5 \sigma$ in their respective centres. 

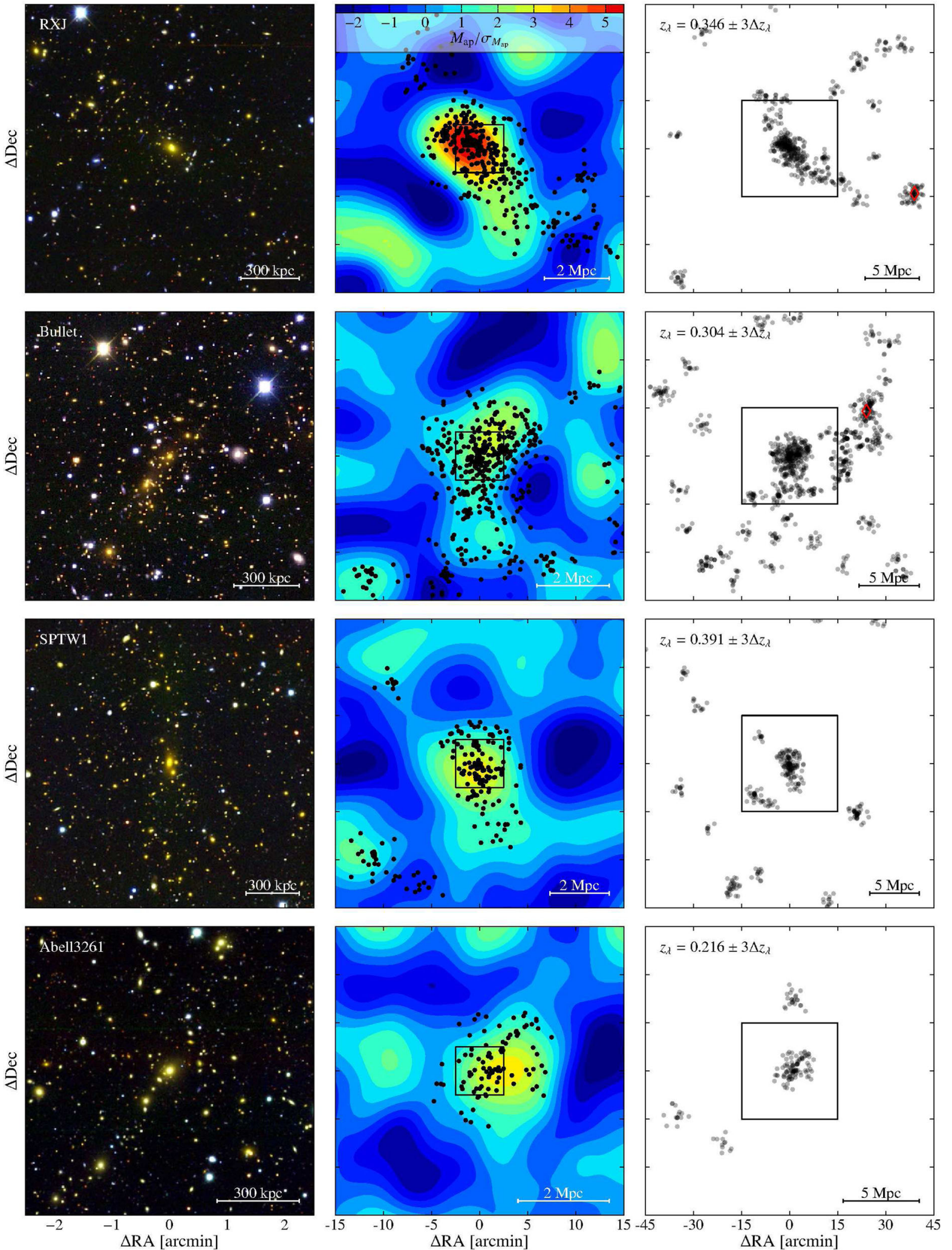

Figure 8. First column: multicolour image of the inner 5 arcmin. Second column: weak-lensing aperture mass significance map of the inner 30 arcmin (contours, cf. equation 19), overlaid with galaxies (black dots) in redMaPPer-detected groups with $\lambda \geq 5$ and redshifts of $z_{\lambda}=z_{\lambda}^{\mathrm{c}} \pm 3 \Delta z_{\lambda}$. Third column: the same redMaPPer galaxies as in the second column, but for the entire useable field of view of 90 arcmin. All panels are centred on the BCGs, the size of the previous (smaller) panel is indicated by black boxes in columns 2 and 3. From top to bottom: RXC J2248.7-4431, 1E 0657-56, SCSO J233227-535827, and Abell 3261. 


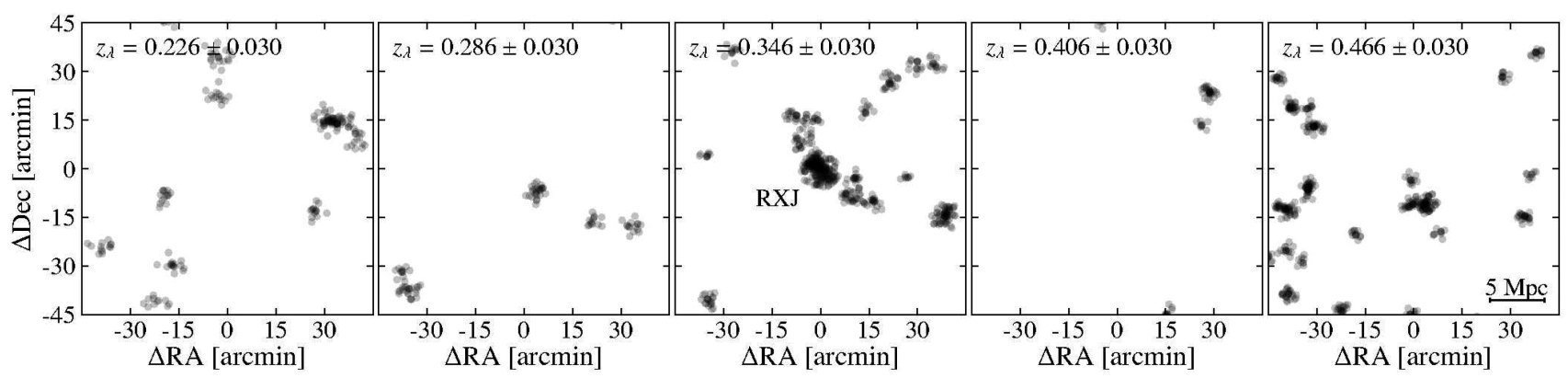

Figure 9. Galaxies in redMaPPer-detected groups in the field of RXC J2248.7-4431 with $\lambda \geq 5$ and redshifts of $z_{\lambda}$ within the indicated non-overlapping redshift slices, centred on the redMaPPer-assigned redshift $z_{\lambda}^{c}=0.344$ of the main cluster.

For the Bullet cluster, the peak significance is not as prominent despite having an expected mass comparable to RXJ, but due to its highly non-spherical mass distribution, the spherical filter shape works against the signal, reducing its amplitude. Finally, even the least massive cluster, Abell 3261, shows up at the level of $3 \sigma$ in its mass map. The reduced significance of the latter two is caused also by a low $n_{\text {gal }} \approx 6 \operatorname{arcmin}^{-2}$ after background cuts.

We overlay the mass maps with redMaPPer-detected galaxies in groups with $\lambda \geq 5$, whose redshift estimates $z_{\lambda}$ are consistent within $\pm 3 \Delta z_{\lambda} \lesssim 0.03$ with the main cluster redshift $z_{\lambda}^{\mathrm{c}}$ (see Section 3.2 for details). The distribution reveals the structure of the red-sequence galaxies within and around the main cluster halo.

Several aspects of the mass maps are remarkable. First, the mass maps clearly follow the red-sequence cluster-galaxy distributions. This is additional confirmation that the shape measurements indeed perform well since we expect mass to trace light. Second, the peaks in the mass maps do not always coincide with the cluster BCGs. For the Bullet cluster, the peak is placed roughly between the main cluster and the subcluster. Given our large smoothing scale, it is not surprising that the two peaks are effectively merged. Furthermore, even for single-peaked mass distributions, such shifts between the BCG and the most prominent mass peak are a consequence of sampling the shear field with a finite number of sources (e.g. Dietrich et al. 2012). Third, we note that additional peaks exist in the mass maps, occasionally reaching $2 \sigma$, that are not associated with the main cluster or other known clusters in the fields. To test the robustness of the mass maps we therefore run bootstrap resamples of the lensed galaxies, which reveal that these peaks are largely spurious and depend on particular configurations of a small number of neighbouring galaxies. In contrast, the peaks associated with the main clusters shift location by up to 2 arcmin but are otherwise robust under resampling.

\subsection{Filamentary features}

In the third column of Fig. 8, we utilize the full DECam image and show the distribution of redMaPPer-detected groups for the entire usable area. We can see that the most massive clusters in the sample show a rich environment that seems connected to the central region and that reaches out to other clusters in the vicinity. From a hierarchical CDM structure formation scenario, we expect such structures, called filaments, to be attached to and to act as bridges between clusters, especially the very massive ones (e.g. Bond, Kofman \& Pogosyan 1996). Both cosmological simulations and spectroscopic surveys have revealed filaments with typical lengths of ten to dozens of Mpc (e.g. Alpaslan et al. 2014; Tempel et al. 2014). Of particular relevance to this study is that in simulations more than 80 per cent of cluster pairs with distances of around $10 \mathrm{Mpc} h^{-1}$ are connected by filaments (Colberg, Krughoff \& Connolly 2005). In the RXJ field, there is another cluster in the same redshift slice, detected by redMaPPer with $\lambda=41 \pm 2$ (red diamond in the top right-hand panel of Fig. 8), at a distance of 40 arcmin to the south-west (12 Mpc in the plane of the sky), and a string of less massive groups that may constitute a mildly curved connecting filament. For the Bullet cluster, we can see another cluster at 28 arcmin to the north-west $(\lambda=78 \pm 3$ ) and other correlated structure over $20 \mathrm{Mpc}$, assuming the entire galaxy distribution is aligned in the plane of the sky - a slight underestimation because it is known that at least the inner region is inclined by $10^{\circ}-15^{\circ}$ (Barrena et al. 2002; Markevitch et al. 2002). Structures that large have only been observed around a few other clusters, mostly at higher redshift than we probe here (e.g. Tanaka et al. 2009; Verdugo et al. 2012).

Without spectroscopic follow-up, we cannot prove that all shown redMaPPer-detected galaxies are indeed at the redshift of the main cluster or gravitationally interacting with the main halo. There are, however, additional aspects that support the notion that the shown structures are indeed real and associated with the clusters. redMaPPer determines the redshifts of the main cluster haloes with high accuracy (compare Table 1 with Table 4), which implies that the overall photometric calibration performs well and that the red-sequence colours are properly calibrated for the DES photometry. This is consistent with the results from Rykoff et al. (in preparation) showing that groups with $\lambda \geq 5$ exhibit scatter of $\sigma_{z_{\lambda}} \leq 0.015$ and negligible bias when compared to existing spectroscopic redshifts of clusters in the DES SV footprint. We can also split the light cone of the observed fields into thin redshift slices to test whether the structures are confined to the redshift of the main cluster halo. The result for the RXJ cluster is shown in Fig. 9. Note that this test is different from the right-hand panels in Fig. 8 in that we do not ask whether the redshift of the group is consistent with the main cluster's at $3 \Delta z_{\lambda}$ (of each group), which potentially allows for an arbitrarily wide redshift range if $\Delta z_{\lambda} \rightarrow \infty$. Instead, we only consider the central value $z_{\lambda}$ of each group and fix the width of the slice at \pm 0.03 , the typical value of $3 \Delta z_{\lambda}$ for groups with $\lambda \geq 5$. This way the influence of chance projections of groups with poorly determined redshifts can be suppressed. We can indeed see that the filamentary structure does not bleed into other slices. Also, apart from additional smaller clusters at different redshifts, the other slices are much less populated and do not show similarly prominent correlated structures. Tests of the other clusters yield similar results.

\section{SUMMARY}

In the study presented here we observed four galaxy cluster fields with the newly installed imager DECam and tested all data 
processing stages necessary for weak lensing applications within the DES. Even with early data observed during the SV phase, we find the instrument and these pipelines to perform according to anticipated specifications and yield astrometry, photometry, and shape measurements adequate for the pathfinder analysis presented here, with no show-stoppers that preclude forthcoming science analyses. Most important in this work was to establish how to obtain reliable shape catalogues from DECam data, and we summarize our findings as follows.

(i) By jointly fitting for the astrometry of all exposures, we find 20 mas scatter in the astrometric solution of SCAMP across the entire focal plane.

(ii) The PSF patterns are spatially fairly smooth across the focal plane and can be well modelled with PSFEx, provided that the brightest stars are discarded to limit the impact of the flux dependence of the PSF width. The majority of the co-add images have PSF model residuals in size and ellipticity that are subdominant compared to shape scatter up to separations of $1^{\circ}$.

(iii) With suitably chosen cuts, IM3SHAPE yields shape measurements with a number density $n_{\text {gal }} \approx 10 \operatorname{arcmin}^{-2}$ as expected for full-depth data at nominal seeing. The results are consistent when varying source flux, size, photo- $z$, or the filter of observation.

These technical prerequisites enable us to utilize the large field of view of DECam to estimate weak lensing masses and to map out the galaxy and mass distribution of the targeted galaxy clusters. Our scientific results are the following.

(i) We find weak lensing masses for RXC J2248.7-4431, the Bullet cluster 1E 0657-56, and SCSO J233227-535827 that are in good agreement with previous studies. For clusters at higher redshift or dedicated high-precision lensing studies of individual systems, deeper imaging than the nominal DES depth of $10 \times 90 \mathrm{~s}$ is advised.

(ii) For the cluster Abell 3261, we provide the first redshift, richness, and weak lensing mass estimates in the literature.

(iii) The mass maps of all four clusters show their most significant peak at or close to the cluster BCG. Clusters with a visibly noticeable alignment of cluster member galaxies exhibit the same orientation also in the mass maps.

(iv) Because of well-calibrated photometry, the red-sequence method redMaPPer detects these four clusters reliably. The redshift estimates of even much smaller clusters with $\lambda>5$ are precise enough (Rykoff et al., in preparation) to form thin slices at the cluster redshift and to map out the distribution of red-sequence galaxies in the entire cluster environment. The most massive systems, 1E 0657-56 and RXC J2248.7-4431, show filamentary structures over about $1^{\circ}$, equivalent to about $20 \mathrm{Mpc}$ at the cluster redshifts. If the presence of these structures can be confirmed, this technique can be employed in the DES main survey and enable efficient searches for large-scale filaments in the entire DES footprint without the need for full spectroscopic coverage.

The work presented here will form the basis of forthcoming analyses within DES, concerning e.g. the cluster mass function, the calibration of mass-observable relations from optical richness, X-ray and SZ effect, and other more demanding lensing applications.

\section{ACKNOWLEDGEMENTS}

We are grateful for the extraordinary contributions of our CTIO colleagues and the DES Camera, Commissioning and Science Verification teams in achieving the excellent instrument and telescope conditions that have made this work possible. The success of this project also relies critically on the expertise and dedication of the DES Data Management organization.

Funding for the DES Projects has been provided by the US Department of Energy, the US National Science Foundation, the Ministry of Science and Education of Spain, the Science and Technology Facilities Council of the United Kingdom, the Higher Education Funding Council for England, the National Center for Supercomputing Applications at the University of Illinois at Urbana-Champaign, the Kavli Institute of Cosmological Physics at the University of Chicago, Financiadora de Estudos e Projetos, Fundação Carlos Chagas Filho de Amparo à Pesquisa do Estado do Rio de Janeiro, Conselho Nacional de Desenvolvimento Científico e Tecnológico and the Ministério da Ciência e Tecnologia, the Deutsche Forschungsgemeinschaft, and the Collaborating Institutions in the Dark Energy Survey.

The Collaborating Institutions are Argonne National Laboratories, the University of California at Santa Cruz, the University of Cambridge, Centro de Investigaciones Energeticas, Medioambientales y Tecnologicas-Madrid, the University of Chicago, University College London, the DES-Brazil Consortium, the Eidgenössische Technische Hochschule (ETH) Zürich, Fermi National Accelerator Laboratory, the University of Edinburgh, the University of Illinois at Urbana-Champaign, the Institut de Ciencies de l'Espai (IEEC/CSIC), the Institut de Fisica d'Altes Energies, the Lawrence Berkeley National Laboratory, the Ludwig-Maximilians Universität and the associated Excellence Cluster Universe, the University of Michigan, the National Optical Astronomy Observatory, the University of Nottingham, the Ohio State University, the University of Pennsylvania, the University of Portsmouth, SLAC National Accelerator Laboratory, Stanford University, the University of Sussex, and Texas A\&M University.

PM, ES, EH, KP, KH are supported by the US Department of Energy under Contract No. DE- FG02-91ER40690. SB, TK, MH, JZ, BR acknowledge support from European Research Council in the form of a Starting Grant with number 240672. DG was supported by SFB-Transregio 33 'The Dark Universe' by the Deutsche Forschungsgemeinschaft (DFG) and the DFG cluster of excellence 'Origin and Structure of the Universe'. The DES participants from Spanish institutions are partially supported by MINECO under grants AYA2009-13936, AYA2012-39559, AYA2012-39620, and FPA2012-39684, which include FEDER funds from the European Union.

This paper has gone through internal review by the DES collaboration.

\section{REFERENCES}

Abell G. O., Corwin H. G., Jr, Olowin R. P., 1989, ApJS, 70, 1

Ahn C. P. et al., 2014, ApJS, 211, 17

Alpaslan M. et al., 2014, MNRAS, 438, 177

Antilogus P., Astier P., Doherty P., Guyonnet A., Regnault N., 2014, J. Instrum., 9, C03048

Applegate D. E. et al., 2014, MNRAS, 439, 48

Barrena R., Biviano A., Ramella M., Falco E. E., Seitz S., 2002, A\&A, 386, 816

Bartelmann M., 1996, A\&A, 313, 697

Bartelmann M., Schneider P., 2001, Phys. Rep., 340, 291

Bernstein G. M., Jarvis M., 2002, AJ, 123, 583

Bertin E., 2006, in Gabriel C., Arviset C., Ponz D., Solano E., eds, ASP Conf. Ser. Vol. 351, Astronomical Data Analysis Software and Systems XV. Astron. Soc. Pac., San Francisco, p. 112 
Bertin E., 2011, in Evans I. N., Accomazzi A., Mink D. J., Rots A. H., eds, ASP Conf. Ser. Vol. 442, Astronomical Data Analysis Software and Systems XX. Astron. Soc. Pac., San Francisco, p. 435

Bertin E., Arnouts S., 1996, A\&AS, 117, 393

Bertin E., Mellier Y., Radovich M., Missonnier G., Didelon P., Morin B., 2002, in Bohlender D. A., Durand D., Handley T. H., eds, ASP Conf. Ser. Vol. 281, Astronomical Data Analysis Software and Systems XI. Astron. Soc. Pac., San Francisco, p. 228

Blazek J., Mandelbaum R., Seljak U., Nakajima R., 2012, J. Cosmol. Astropart. Phys., 5, 41

Böhringer H. et al., 2004, A\&A, 425, 367

Bond J. R., Kofman L., Pogosyan D., 1996, Nature, 380, 603

Bullock J. S., Kolatt T. S., Sigad Y., Somerville R. S., Kravtsov A. V., Klypin A. A., Primack J. R., Dekel A., 2001, MNRAS, 321, 559

Carter D., Metcalfe N., 1980, MNRAS, 191, 325

Chisari N. E., Mandelbaum R., Strauss M. A., Huff E. M., Bahcall N. A., 2014, MNRAS, 445, 726

Clowe D., Gonzalez A., Markevitch M., 2004, ApJ, 604, 596

Colberg J. M., Krughoff K. S., Connolly A. J., 2005, MNRAS, 359, 272

Desai S. et al., 2012, ApJ, 757, 83

Diehl T., 2012, Phys. Proc., 37, 1332

Dietrich J. P., Böhnert A., Lombardi M., Hilbert S., Hartlap J., 2012 MNRAS, 419, 3547

Duffy A. R., Schaye J., Kay S. T., Dalla Vecchia C., 2008, MNRAS, 390, L64

Flaugher B. L. et al., 2012, Proc. SPIE, 8446, 844611

Foreman-Mackey D., Hogg D. W., Lang D., Goodman J., 2013, PASP, 125 306

Górski K. M., Hivon E., Banday A. J., Wandelt B. D., Hansen F. K., Reinecke M., Bartelmann M., 2005, ApJ, 622, 759

Gruen D. et al., 2013, MNRAS, 432, 1455

Gruen D., Seitz S., Bernstein G. M., 2014a, PASP, 126, 158

Gruen D. et al., 2014b, MNRAS, 442, 1507

Hao J., Kubo J. M., Feldmann R., Annis J., Johnston D. E., Lin H., McKay T. A., 2011, ApJ, 740, 39

Heymans C. et al., 2012, MNRAS, 427, 146

High F. W., Stubbs C. W., Rest A., Stalder B., Challis P., 2009, AJ, 138, 110

High F. W. et al., 2010, ApJ, 723, 1736

Hoekstra H., Franx M., Kuijken K., 2000, ApJ, 532, 88

Hoekstra H., Mahdavi A., Babul A., Bildfell C., 2012, MNRAS, 427, 1298

Høg E. et al., 2000, A\&A, 355, L27

Kacprzak T., Zuntz J., Rowe B., Bridle S., Refregier A., Amara A., Voigt L., Hirsch M., 2012, MNRAS, 427, 2711

Kelly P. L. et al., 2014, MNRAS, 439, 28

Kitching T. D. et al., 2012, MNRAS, 423, 3163

Koester B. P. et al., 2007, ApJ, 660, 239

Mandelbaum R., Hirata C. M., Leauthaud A., Massey R. J., Rhodes J., 2012, MNRAS, 420, 1518

Manfroid J., 1995, A\&AS, 113, 587

Markevitch M., Gonzalez A. H., David L., Vikhlinin A., Murray S., Forman W., Jones C., Tucker W., 2002, ApJ, 567, L27

Massey R. et al., 2007, MNRAS, 376, 13

Maturi M., Meneghetti M., Bartelmann M., Dolag K., Moscardini L., 2005, A\&A, 442, 851

Medezinski E. et al., 2013, ApJ, 777, 43

Melchior P., Viola M., 2012, MNRAS, 424, 2757

Melchior P., Viola M., Schäfer B. M., Bartelmann M., 2011, MNRAS, 412, 1552

Menanteau F. et al., 2010a, ApJS, 191, 340

Menanteau F. et al., 2010b, ApJ, 723, 1523

Merten J. et al., 2011, MNRAS, 417, 333

Merten J. et al., 2014, ApJ, preprint (arXiv:1404.1376)

Miller L. et al., 2013, MNRAS, 429, 2858

Moffat A. F. J., 1969, A\&A, 3, 455

Mohr J. J. et al., 2012, Proc. SPIE, 8451, 84510D

Navarro J. F., Frenk C. S., White S. D. M., 1996, ApJ, 462, 563

Neilsen E., Annis J., 2014, in Manset N., Forshay P., eds, ASP Conf. Ser. Vol. 485, Astronomical Data Analysis Software and Systems XXIII. Astron. Soc. Pac., San Francisco, p. 77
Oguri M., Hamana T., 2011, MNRAS, 414, 1851

Oyaizu H., Lima M., Cunha C. E., Lin H., Frieman J., Sheldon E. S., 2008a, ApJ, 674, 768

Oyaizu H., Lima M., Cunha C. E., Lin H., Frieman J., 2008b, ApJ, 689, 709 Paulin-Henriksson S., Amara A., Voigt L., Refregier A., Bridle S. L., 2008, A\&A, 484, 67

Pickles A. J., 1998, PASP, 110, 863

Postman M. et al., 2012, ApJS, 199, 25

Refregier A., Kacprzak T., Amara A., Bridle S., Rowe B., 2012, MNRAS, 425, 1951

Reichardt C. L. et al., 2013, ApJ, 763, 127

Rowe B., 2010, MNRAS, 404, 350

Rowe B. et al., 2014, Astron. Comput., preprint (arXiv:1407.7676)

Rozo E., Rykoff E. S., 2014, ApJ, 783, 80

Rykoff E. S. et al., 2012, ApJ, 746, 178

Rykoff E. S. et al., 2014, ApJ, 785, 104

Sánchez C. et al., 2014, MNRAS, 445, 1482

Sastry G. N., 1968, PASP, 80, 252

Schlegel D. J., Finkbeiner D. P., Davis M., 1998, ApJ, 500, 525

Schneider P., 1996, MNRAS, 283, 837

Sérsic J. L., 1963, Bol. Asociacion Argentina Astron., 6, 41

Sifón C., Hoekstra H., Cacciato M., Viola M., Köhlinger F., van der Burg R. F. J., Sand D. J., Graham M. L., 2015, A\&A, 575, A48

Tanaka M., Finoguenov A., Kodama T., Koyama Y., Maughan B., Nakata F., 2009, A\&A, 505, L9

Tempel E., Stoica R. S., Martínez V. J., Liivamägi L. J., Castellan G., Saar E., 2014, MNRAS

The Dark Energy Survey Collaboration, 2005, preprint (arXiv:astro-ph/0510346)

Tucker W. et al., 1998, ApJ, 496, L5

Tucker D. L. et al., 2007, in Sterken C., ed., ASP Conf. Ser. Vol. 364, The Future of Photometric, Spectrophotometric and Polarimetric Standardization. Astron. Soc. Pac., San Francisco, p. 187

Umetsu K. et al., 2014, ApJ, 795, 163

Verdugo M., Lerchster M., Böhringer H., Hildebrandt H., Ziegler B. L., Erben T., Finoguenov A., Chon G., 2012, MNRAS, 421, 1949

Wright C. O., Brainerd T. G., 2000, ApJ, 534, 34

Zacharias N., Finch C. T., Girard T. M., Henden A., Bartlett J. L., Monet D. G., Zacharias M. I., 2013, AJ, 145, 44

Zuntz J., Kacprzak T., Voigt L., Hirsch M., Rowe B., Bridle S., 2013, MNRAS, 434, 1604

\section{APPENDIX A: PSF FLUX DEPENDENCE}

The most important test for the PSF model is whether it can reproduce the sizes and ellipticities of observed stars in the field. When using the full range of stellar fluxes to inform the PSF model, we unfortunately register that the PSF width $s$ is overestimated for the majority of all stars (see left-hand panel of Fig. A1). This is a direct consequence of the flux-dependent charge registration in the DECam CCDs, for which we currently do not yet have a chip-level correction. There is furthermore a broadening of the stellar ellipticity residuals, foremost in the $\epsilon_{1}$ direction, the cause of which is not fully understood.

Irrespective of the actual mechanism at work, we can effectively reduce the impact of the flux dependency by excluding the brightest stars when computing the PSF model. We found that rejecting the brightest 3 mag below saturation level, corresponding roughly to MAG_AUTO $\in[15,18]$, allows for very accurate PSF models as determined by the diagnostics in equation (11) and exemplified in Fig. 3, where we used the fainter star selection for the same field as in Fig. A1. While there will be a slight misestimate of the effective PSF a galaxy at our faintest magnitudes of $i \simeq 24.5$ would encounter, the change in flux and therefore in size compared to our fainter star selection is too small to be detectable by the diagnostics employed in this paper. As a practical consequence, we need to 

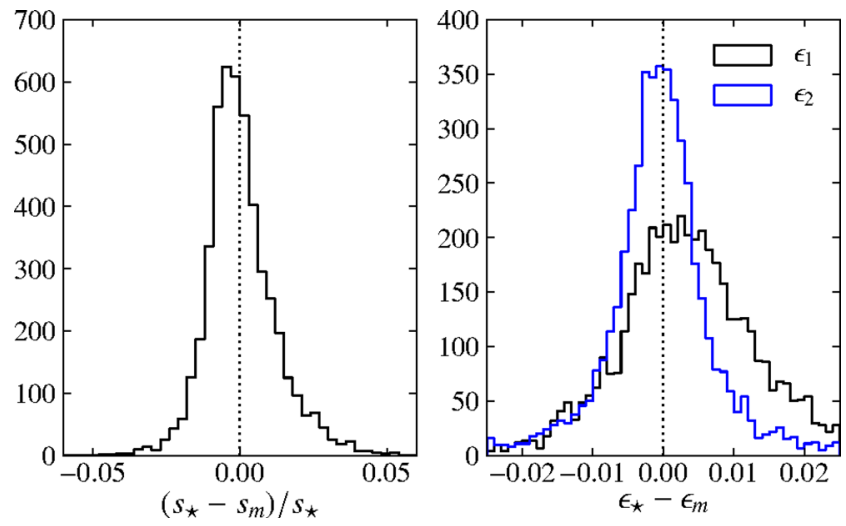

Figure A1. Fractional size and ellipticity residuals of the PSFEX model shown in Fig. 3 but for a set of stars with MAG_AUTO $\in[15,21.5]$. The brighter stars in this selection lead the PSF model to adopt larger sizes and a preferred $\epsilon_{1}$-direction, which is not shared by the bulk of the (fainter) stars.

work with co-added images where the fainter stars can reliably be discriminated from galaxies.

\section{APPENDIX B: TOLERANCES FOR PSF MODEL DIAGNOSTICS}

In this section we seek to propagate failures of the PSF modelling approach in capturing both sizes and ellipticities of the actual PSF (and its spatial variation) into the shear catalogues, thereby establishing limits on the required accuracy of the PSF models. We start with equation (13) from Paulin-Henriksson et al. (2008),

$\Delta \epsilon_{\mathrm{sys}} \simeq\left(\epsilon_{\mathrm{gal}}-\epsilon_{\star}\right) \frac{\Delta\left(s^{2}\right)}{s_{\mathrm{gal}}^{2}}-\left(\frac{s_{\star}}{s_{\mathrm{gal}}}\right)^{2} \Delta \epsilon$,

which estimates the systematic error in the shape of a deconvolved galaxy from the uncertainties in size and ellipticity of the PSF model, $\Delta\left(s^{2}\right)$ and $\Delta \epsilon .^{22}$ Forming the correlation function yields

$$
\begin{aligned}
\left\langle\Delta \epsilon_{\mathrm{sys}}^{*} \Delta \epsilon_{\mathrm{sys}}\right\rangle= & {\left[\sigma_{\epsilon}^{2}+\left\langle\epsilon_{\star}^{*} \epsilon_{\star}\right\rangle\right]\left\langle\left(\frac{\Delta s^{2}}{s_{\text {gal }}^{2}}\right)^{2}\right\rangle+\left\langle\left(\frac{s_{\star}}{s_{\text {gal }}}\right)^{4}\right\rangle } \\
& \times\left\langle\Delta \epsilon^{*} \Delta \epsilon\right\rangle+\left\langle\frac{\Delta s^{2} s_{\star}^{2}}{s_{\text {gal }}^{4}}\left(\epsilon_{\star}^{*} \Delta \epsilon+\Delta \epsilon^{*} \epsilon_{\star}\right)\right\rangle .
\end{aligned}
$$

Now we will have to make several assumptions to relate the terms arising here to the PSF model diagnostics defined in equations (9) and (10). First, to pull out a common size ratio for the first two terms, we need to assume that the size residuals do not correlate with stellar size:

$$
\begin{aligned}
\left\langle\Delta \epsilon_{\mathrm{sys}}^{*} \Delta \epsilon_{\mathrm{sys}}\right\rangle= & \left\langle\left(\frac{s_{\star}}{s_{\mathrm{gal}}}\right)^{4}\right\rangle\left[\left[\sigma_{\epsilon}^{2}+\left\langle\epsilon_{\star}^{*} \epsilon_{\star}\right\rangle\right] \rho_{3}+\rho_{1}\right] \\
& +\left\langle\frac{\Delta s^{2} s_{\star}^{2}}{s_{\mathrm{gal}}^{4}}\left(\epsilon_{\star}^{*} \Delta \epsilon+\Delta \epsilon^{*} \epsilon_{\star}\right)\right\rangle .
\end{aligned}
$$

\footnotetext{
${ }^{22}$ While the derivation strictly applies to moment-based measures only, and we seek to use it in a model-fitting context, the results depend weakly on the method used for shear estimation, provided the method yields an shape estimate that transforms as an ellipticity under an applied shear.
}

If we furthermore assume that $\Delta \epsilon$ and $\epsilon_{\star}$ as well as $\Delta s^{2}$ and $\Delta \epsilon$ are uncorrelated, we can simplify the last term to

$$
\left\langle\frac{\Delta s^{2} s_{\star}^{2}}{s_{\mathrm{gal}}^{4}}\right\rangle\left\langle\epsilon_{\star}^{*} \Delta \epsilon+\Delta \epsilon^{*} \epsilon_{\star}\right\rangle,
$$

which allows us to identify it with $\rho_{2}$ :

$$
\left\langle\Delta \epsilon_{\text {sys }}^{*} \Delta \epsilon_{\text {sys }}\right\rangle=\left\langle\left(\frac{s_{\star}}{s_{\text {gal }}}\right)^{4}\right\rangle\left[\left[\sigma_{\epsilon}^{2}+\left\langle\epsilon_{\star}^{*} \epsilon_{\star}\right\rangle\right] \rho_{3}+\rho_{1}+\left\langle\frac{\Delta s^{2}}{s_{\star}^{2}}\right\rangle \rho_{2}\right] .
$$

We note that these additional assumptions are clearly problematic as one could easily imagine that residuals increase when the quantity that is modelled increases. For instance, both sizes and ellipticities tend to rapidly rise towards the field edges, where only a small number of stars can constrain the PSF model, a situation that should result in larger and correlated residuals for size and ellipticity.

Ideally, one would assess PSF model errors directly from equation (B2), which considers all possible correlation between sizes and ellipticities (and their errors). We leave this to a forthcoming investigation and want to highlight another immediate consequence of our derivation. If we accept the limitations laid out above resulting in equation (B5), we see that for $\rho_{3}$ a pre-factor of order $10^{-2}$ and for $\rho_{2}$ of order $10^{-3}$ (for a reasonably well-fit PSF model) reduces their relative impact on the total shape error. In other words, if all diagnostic correlation functions were equal, $\rho_{1}$ is most demanding, followed by $\rho_{3}$ and then $\rho_{2}$. In practice, we find relatively larger size than ellipticity residuals, rendering $\rho_{3}$ a useful and, in the case of the flux-dependent PSF, even decisive diagnostic. On the other hand, due to its very small pre-factor, $\rho_{2}$ appears not to carry substantial information to assess the PSF model quality. We will therefore refrain from enforcing limits on $\rho_{2}$ and will assess the PSF models with $\rho_{1}$ and $\rho_{3}$ only.

The left-hand side of equation (B5) differs from the shear estimate only by the shear responsivity $P_{\gamma}$, which allows us to compare the total systematic budget with the statistical limit from the intrinsic shape scatter of the galaxies. For the two-point function, the number of galaxy pairs in a distance bin around $r$ is given by $n_{\text {gal }} \pi\left(R_{\max }^{2}-\right.$ $R_{\min }^{2}$ ), with $R_{\min / \max }$ denoting the minimum and maximum radius of that bin. Assuming a Gaussian form of the intrinsic shape scatter with variance $\sigma_{\epsilon}^{2}$, we get

$P_{\gamma}^{-2}\left\langle\Delta \epsilon_{\mathrm{sys}}^{*} \Delta \epsilon_{\mathrm{sys}}\right\rangle<\frac{\sigma_{\epsilon}^{2}}{n_{\mathrm{gal}} \pi\left(R_{\max }^{2}-R_{\min }^{2}\right)}$.

We still need an estimate for the pre-seeing size of galaxies $s_{\text {gal }}$ in our shape catalogues. For Gaussian-shaped galaxies and stars, one can directly relate the measurement of FWHM_RATIO from IM3SHAPE to the ratio of the moment-based size definition $s$ we have adopted in this paper:

$\frac{s_{\text {gal }}^{2}}{s_{\star}^{2}}=$ FWHM $\_$RATIO ${ }^{2}-1$.

Together with the shear responsivity yields

$T=P_{\gamma} \frac{s_{\text {gal }}^{2}}{s_{\star}^{2}}=P_{\gamma}\left(\right.$ FWHM_RATIO $\left.{ }^{2}-1\right)$.

Finally, requiring that no diagnostic function alone crosses the limit set by equation (B6), we get the set of tolerances in equation (11).

${ }^{1}$ Center for Cosmology and Astro-Particle Physics, The Ohio State University, Columbus, OH 43210, USA 
${ }^{2}$ Department of Physics, The Ohio State University, Columbus, $\mathrm{OH} 43210$, USA

${ }^{3}$ Department of Physics and Astronomy, University College London, Gower Street, London WC1E 6BT, UK

${ }^{4}$ Jodrell Bank Center for Astrophysics, School of Physics and Astronomy, University of Manchester, Oxford Road, Manchester M13 9PL, UK

${ }^{5}$ SLAC National Accelerator Laboratory, Menlo Park, CA 94025, USA

${ }^{6}$ University Observatory Munich, Scheinerstrasse 1, D-81679 Munich, Germany

${ }^{7}$ Max Planck Institute for Extraterrestrial Physics, Giessenbachstrasse, D-85748 Garching, Germany

${ }^{8}$ Department of Physics and Astronomy, University of Pennsylvania, Philadelphia, PA 19104, USA

${ }^{9}$ Institute of Cosmology and Gravitation, University of Portsmouth, Portsmouth PO1 3FX, UK

${ }^{10}$ Kavli Institute for Cosmological Physics, University of Chicago, Chicago, IL 60637, USA

${ }^{11}$ Fermi National Accelerator Laboratory, P.O. Box 500, Batavia, IL 60510, USA

${ }^{12}$ Brookhaven National Laboratory, Bldg 510, Upton, NY 11973, USA

${ }^{13}$ Cerro Tololo Inter-American Observatory, National Optical Astronomy Observatory, Casilla 603, La Serena, Chile

${ }^{14}$ Space Telescope Science Institute, 3700 San Martin Drive, Baltimore, MD 21218, USA

${ }^{15}$ Argonne National Laboratory, 9700 South Cass Avenue, Lemont, IL 60439, USA

${ }^{16}$ Carnegie Observatories, 813 Santa Barbara St., Pasadena, CA 91101, USA

${ }^{17}$ Institut d'Astrophysique de Paris, Univ. Pierre et Marie Curie and CNRS UMR7095, F-75014 Paris, France

${ }^{18}$ Kavli Institute for Particle Astrophysics and Cosmology, 452 Lomita Mall, Stanford University, Stanford, CA 94305, USA

${ }^{19}$ Institut de Ciències de l'Espai, IEEC-CSIC, Campus UAB, Facultat de Ciències, Torre C5 par-2, E-08193 Bellaterra, Barcelona, Spain

${ }^{20}$ Observatório Nacional, Rua Gal. José Cristino 77, 20921-400 Rio de Janeiro, RJ, Brazil
${ }^{21}$ Laboratório Interinstitucional de e-Astronomia - LIneA, Rua Gal. José Cristino 77, 20921-400 Rio de Janeiro, RJ, Brazil

${ }^{22}$ George P. and Cynthia Woods Mitchell Institute for Fundamental Physics and Astronomy, and Department of Physics and Astronomy, Texas A\&M University, College Station, TX 77843, USA

${ }^{23}$ Department of Physics, Ludwig-Maximilians-Universität, Scheinerstr. 1, D-81679 München, Germany

${ }^{24}$ Excellence Cluster Universe, Boltzmannstr. 2, D-85748 Garching, Germany

${ }^{25}$ Department of Physics, University of Michigan, Ann Arbor, MI 48109, USA

${ }^{26}$ Department of Astronomy, University of Michigan, Ann Arbor, MI 48109, USA

${ }^{27}$ Institut de Física d'Altes Energies, Universitat Autònoma de Barcelona, E-08193 Bellaterra, Barcelona, Spain

${ }^{28}$ Department of Astronomy, University of Illinois, $1002 \mathrm{~W}$. Green Street, Urbana, IL 61801, USA

${ }^{29}$ National Center for Supercomputing Applications, 1205 West Clark St., Urbana, IL 61801, USA

${ }^{30}$ Department of Physics, University of Illinois, 1110 W. Green St., Urbana, IL 61801, USA

${ }^{31}$ Australian Astronomical Observatory, North Ryde, NSW 2113, Australia

${ }^{32}$ ICRA, Centro Brasileiro de Pesquisas Físicas, Rua Dr. Xavier Sigaud 150, CEP 22290-180, Rio de Janeiro, RJ, Brazil

${ }^{33}$ Institució Catalana de Recerca i Estudis Avançats, E-08010 Barcelona, Spain

${ }^{34}$ Lawrence Berkeley National Laboratory, 1 Cyclotron Road, Berkeley, CA 94720, USA

${ }^{35}$ Centro de Investigaciones Energéticas, Medioambientales y Tecnológicas (CIEMAT), E-28040 Madrid, Spain

${ }^{36}$ Instituto de Física, UFRGS, Caixa Postal 15051, 91501-970 Porto Alegre, RS, Brazil

${ }^{37}$ SEPnet, South East Physics Network, UK (www.sepnet.ac.uk)

This paper has been typeset from a $\mathrm{T}_{\mathrm{E}} \mathrm{X} / \mathrm{L}_{\mathrm{E}} \mathrm{X}$ file prepared by the author. 